\title{
An $h p$ symplectic pseudospectral method for nonlinear optimal control
}

\author{
Haijun Peng*; Xinwei Wang; Mingwu Li; Biaosong Chen \\ Department of Engineering Mechanics, State Key Laboratory of Structural Analysis for Industrial \\ Equipment, Dalian University of Technology, Dalian, Liaoning 116024, China
}

\begin{abstract}
:
An adaptive symplectic pseudospectral method based on the dual variational principle is proposed and is successfully applied to solving nonlinear optimal control problems in this paper. The proposed method satisfies the first order necessary conditions of continuous optimal control problems, also the symplectic property of the original continuous Hamiltonian system is preserved. The original optimal control problem is transferred into a set of nonlinear equations which can be solved easily by Newton-Raphson iterations, and the Jacobian matrix is found to be sparse and symmetric. The proposed method, on one hand, exhibits exponent convergence rates when the number of collocation points are increasing with the fixed number of sub-intervals; on the other hand, exhibits linear convergence rates when the number of sub-intervals is increasing with the fixed number of collocation points. Furthermore, combining with the $h p$ method based on the residual error of dynamic constraints, the proposed method can achieve given precisions in a few iterations. Five examples highlight the high precision and high computational efficiency of the proposed method.
\end{abstract}

Keywords: nonlinear optimal control; symplectic; pseudospectral method; variational principles; $h p$ method; Hamiltonian system

*Corresponding author E-mail: hjpeng@dlut.edu.cn. 


\section{Introduction}

As an important part of nonlinear science and engineering, the technique of nonlinear optimal control theories and methods has been widely used in diverse engineering fields, such as chemical engineering [1-3], vibration engineering [4-5], robotics [6-8] and astrodynamics [9-12]. It is extremely difficult to analytically solve a nonlinear optimal control problem. Thus numerous computational techniques for solving nonlinear optimal control problems have been developed in the literature, and they fall into two general categories [13-14]: direct methods and indirect methods.

In an indirect method, the original nonlinear optimal control problem is transformed into a nonlinear two-point boundary value problem (TPBVP) using the first order necessary conditions for optimal control problems [13]. The main advantages of indirect methods come down to two aspects. On one hand, once a numerical solution is obtained then it naturally becomes a locally optimal solution since first order necessary conditions are used. On the other hand, as the solution of most indirect methods includes the information of costate variables, together with the value of state variables it can be used to analyze the characteristic of geometric structures of the Hamiltonian dynamic system. However, different kinds of constrains are required to be handled specifically according to the type of constraints. Various numerical methods such as the shooting method [15-17], the multiple shooting method [18], the generating function method [19] and the finite difference method [20] can be adopted to solve TPBVPs. For shooting methods, initial guesses of unknown state and/or costate variables which must satisfy transversality conditions are required. They can achieve effective convergence but may cause ill-conditioning for problems with a long time interval. In generating function methods, guesses of initial or terminal costate variables are not needed which is an advantage over the shooting method. However, complicated series expansions and lots of ordinary differential equations are required in generating function methods.

In contrast to indirect methods, direct methods try to optimize the cost functional directly. In a direct method, the nonlinear optimal control problem is transformed into a finite dimensional nonlinear programming problem (NLP) [13]. Various types of constraints can be treated in a uniform framework [21] in direct methods which is an advantage over indirect methods. Over past two decades, direct collocation nonlinear programming program (DCNLP) methods have become popular. In DCNLP methods, state and control variables are approximated by a set of trial functions and the dynamic 
constraints are collocated at specified set of points in the solution domain. More recently, pseudospectral methods have attracted much attention. In most pseudospectral methods, the collocation points are based on Gaussian quadrature rules and the basic functions are commonly Legendre or Chebyshev polynomials. According to the scheme of interpolation, various kinds of interpolation points such as Chebyshev-Gauss-Lobatto (CGL) [22], Legendre-Gauss (LG) [23-24], Legendre-Gauss-Radau (LGR) [25-28] and Legendre-Gauss-Lobatto (LGL) [29] can be obtained. Estimations of costate variables cannot be obtained in the majority of direct methods so that it is impossible to determine whether those methods satisfy the first order necessary conditions for optimal control problems. Nevertheless, it has been proved that the Karush-Kuhn-Tucker (KKT) conditions in pseudospectral methods are equivalent to the first order necessary conditions for optimal control problems. For problems whose solutions are smooth and well behaved, pseudospectral methods have a simple structure and converge at an exponential rate. However, the exponential convergence rate may lose when solutions are non-smooth. Due to these advantages, pseudospectral methods have been widely used in aeronautics and astronautics and a software package, i.e., GPOPS [26] based on MATLAB is developed. More recently, Mardsen and his coworkers proposed discrete mechanics and optimal control (DMOC) [30] which belongs to direct methods using discretization. In DMOC state equations are discretized based on the Lagrange-d'Alembert principle, which plays an important role in theoretical mechanics.

The optimal control problems can be transferred into Hamiltonian system by the variational principle or the Pontryagin's maximum principle. The most fundamental property of Hamiltonian systems is that the phase flow is a symplectic transformation [31]. Thus numerical methods that preserve the symplectic structure are more effective and accurate for solving Hamiltonian systems. In fact, generating function methods and the DMOC method mentioned above are both symplectic algotithms. Recent years, Peng and his coworkers proposed a series of symplecitc methods where state and costate variables within a time interval are approximated by using Lagrange polynomial and variables at two time ends of the time interval are taken as independent variables [32-36]. According to different choices of independent variables, four different kinds of action are proposed.

Originally, the DCNLP methods mentioned above are developed as $h$ methods, where the whole time domain is divided into some small sub-intervals and state and/or control variables are 
approximated using fixed order of interpolation function in each sub-interval. Then, convergences in $h$ methods are achieved by increasing the number of sub-intervals and/or replacing the position of sub-intervals. While primarily, pseudospectral methods are considered to be $p$ methods, where the approximation of state and/or control variables are implemented in the whole time domain which is treated as a single mesh interval. Convergences in $p$ methods are thus accomplished by increasing the degree of approximation polynomial. Yet, both $h$ methods and $p$ methods are born with limitations. Specifically, it may lead to an extremely fine mesh (in $h$ methods) or an unreasonably high degree of interpolation function (in $p$ methods) for achieving the given accuracy. In order to combine the characteristic of $h$ methods and $p$ methods, $h p$ methods, where both the number of sub-intervals and the degree of approximation polynomials are allowed to change, have been developed [24,27,28,37]. $H p$ methods are originally developed for solving partial differential equations [38-41] and applied to solving optimal control problems in recent years. In this paper, an $h p$ adaptive method based on the residual error of dynamic equations is developed.

Since symplectic-preserving algorithms are highly efficient, and pseudospectral methods exhibit the exponent rate of convergence for problems where solutions are smooth, a symplectic pseudospectral method is attractive. Thus, an adaptive symplectic pseudospectral method for solving nonlinear optimal control problems is proposed in this paper. The original optimal control problem is transformed into a nonlinear TPBVP using the first order necessary conditions for optimal control problems. The whole time domain is divided into several sub-intervals, and the action within each sub-interval is approximated by the LGL quadrature scheme. Thus state and costate variables are naturally discretized at LGL points and approximated by Lagrangian interpolation functions. Based on the dual variational principle and taking state variables at two ends of a sub-interval as independent variables, the nonlinear TPBVP is transformed into a set of nonlinear equations. After applying boundary conditions, the set of nonlinear equations can be solved easily by Newton-Raphson iteration methods. The Jacobian matrix in the Newton iterations is found to be sparse and symmetric, and then lead to a high computational efficiency and high precision. Initial guesses in the Newton iterations are not required to satisfy transversality conditions and final solutions are not sensitive to initial guesses. Combining with an $h p$ adaptive procedure based on residual error of dynamic constraints, numerical solutions of given precision can be obtained in a few iterations. 


\section{Problem formulation}

Considering the following nonlinear optimal control problem, the main objective of optimal control is to determine the state variables $\mathbf{x} \in \mathbb{R}^{d}$ and the control input $\mathbf{u} \in \mathbb{R}^{p}$ within the time interval $t \in\left[0, t_{f}\right]$ to minimize the following Bolza cost functional

$$
J=h\left(\mathbf{x}\left(t_{f}\right), t_{f}\right)+\int_{0}^{t_{f}} \Phi(\mathbf{x}(t), \mathbf{u}(t), t) d t
$$

Meanwhile, subjecting to the dynamic differential equations

$$
\dot{\mathbf{x}}=\mathbf{f}(\mathbf{x}(t), \mathbf{u}(t), t), \quad \mathbf{x}(0)=\mathbf{x}_{0}
$$

where the dot represents the derivative with respect to time, and $\mathbf{x}_{0}$ is the initial condition for the state variables. As for the boundary condition at the terminal time, either the fixed terminal state or the free terminal state is feasible. For simplicity, this paper considers optimal control problems with a fixed terminal time (i.e., $t_{f}$ is given).

By introducing Lagrangian multipliers $\lambda$ which is also called costate variables in optimal control problems, the dynamic differential equations can be eliminated and a new augmented cost functional is obtained as follow:

$$
J_{A}=h\left(\mathbf{x}\left(t_{f}\right), t_{f}\right)+\int_{0}^{t_{f}}\left[\Phi(\mathbf{x}, \mathbf{u}, t)+\lambda^{\mathrm{T}}(\mathbf{f}(\mathbf{x}, \mathbf{u}, t)-\dot{\mathbf{x}})\right] d t
$$

Then the Hamiltonian function $\bar{H}(\mathbf{x}, \mathbf{u}, \lambda, t)$ is introduced as follow

$$
\bar{H}(\mathbf{x}, \mathbf{u}, \lambda, t)=\Phi(\mathbf{x}, \mathbf{u}, t)+\lambda^{\mathrm{T}} \mathbf{f}(\mathbf{x}, \mathbf{u}, t)
$$

The necessary conditions for the optimal control input can be obtained by variational methods as

$$
\frac{\partial \bar{H}(\mathbf{x}, \lambda, \mathbf{u}, t)}{\partial \mathbf{u}}=\mathbf{0}
$$

It is assumed that the optimal control input can be explicitly expressed as a function of state and costate variables. Then the expression of the control input can be obtained by solving Eq. (5) as

$$
\mathbf{u}=\mathbf{u}(\mathbf{x}, \lambda, t)
$$

Substituting Eq. (6) into Eq. (4), a new Hamiltonian function $H(\mathbf{x}, \boldsymbol{\lambda}, t)$ which is only dependent on state variables and costate variables can be obtained and the augmented cost functional $J_{A}$ can be rewritten as 


$$
J_{A}=h\left(\mathbf{x}\left(t_{f}\right), t_{f}\right)+\int_{0}^{t_{f}}\left[H(\mathbf{x}, \boldsymbol{\lambda}, t)-\lambda^{\mathrm{T}} \dot{\mathbf{x}}\right] d t
$$

The variational computation of the cost functional yields

$$
\delta J_{A}=\frac{\partial h\left(\mathbf{x}\left(t_{f}\right), t_{f}\right)}{\partial \mathbf{x}\left(t_{f}\right)} \delta \mathbf{x}\left(t_{f}\right)-\left.\left(\lambda^{\mathrm{T}} \delta \mathbf{x}\right)\right|_{0} ^{t_{f}}+\int_{0}^{t_{f}}\left[\left(\dot{\mathbf{x}}-\frac{\partial H}{\partial \boldsymbol{\lambda}}\right) \delta \boldsymbol{\lambda}+\left(\dot{\boldsymbol{\lambda}}+\frac{\partial H}{\partial \mathbf{x}}\right) \delta \mathbf{x}\right] d t=0
$$

From Eq. (8), it is noted that the optimal control problem has been transferred into a TPBVP, i.e,

$$
\begin{gathered}
\dot{\mathbf{x}}=\frac{\partial H}{\partial \lambda} \\
\dot{\lambda}=-\frac{\partial H}{\partial \mathbf{x}}
\end{gathered}
$$

The state and costate variables at the terminal time are denoted as $\mathbf{x}_{f}$ and $\boldsymbol{\lambda}_{f}$, respectively. Then the boundary conditions can be derived from Eq. (8). i.e.,

$$
\begin{aligned}
& \text { fixed terminal state: } \mathbf{x}(0)=\mathbf{x}_{0}, \mathbf{x}\left(t_{f}\right)=\mathbf{x}_{f} \\
& \text { free terminal state: } \mathbf{x}(0)=\mathbf{x}_{0}, \lambda\left(t_{f}\right)=\frac{\partial h\left(\mathbf{x}\left(t_{f}\right), t_{f}\right)}{\partial \mathbf{x}\left(t_{f}\right)}
\end{aligned}
$$

Shooting methods can be adopted to solve TPBVPs, yet they may cause ill-conditioning for problems with long time span. In the following sections, an adaptive symplectic pseudospectral method is proposed. Compared with shooting methods, the proposed method can get highly precise solutions by smaller amount of iterations and in less computational time.

\section{Symplectic pseudospectral methods based on the dual variational principle}

\subsection{Constructing the symplectic-preserving approach}

The symplecitc pseudospectral method proposed in this paper is based on the dual variational principle. We define a dual action $S$ within time interval $\left[0, t_{f}\right]$ as

$$
S=\int_{0}^{t_{f}}\left[\lambda^{\mathrm{T}} \dot{\mathbf{x}}-H(\mathbf{x}, \boldsymbol{\lambda}, t)\right] d t
$$

By taking variation of Eq. (13), it yields

$$
\delta S=\lambda^{\mathrm{T}}\left(t_{f}\right) \delta \mathbf{x}\left(t_{f}\right)-\boldsymbol{\lambda}^{\mathrm{T}}(0) \delta \mathbf{x}(0)+\int_{0}^{t_{f}}\left[\left(\dot{\mathbf{x}}-\frac{\partial H}{\partial \boldsymbol{\lambda}}\right)^{\mathrm{T}} \delta \boldsymbol{\lambda}-\left(\dot{\boldsymbol{\lambda}}+\frac{\partial H}{\partial \mathbf{x}}\right)^{\mathrm{T}} \delta \mathbf{x}\right] d t
$$

It is seen that the Hamiltonian canonical equations shows in Eqs. (9) (10) can be obtained by the variation of the action $S$. Additionally, if the Hamiltonian canonical equations are satisfied, one has 


$$
\delta S=\lambda^{\mathrm{T}}\left(t_{f}\right) \delta \mathbf{x}\left(t_{f}\right)-\lambda^{\mathrm{T}}(0) \delta \mathbf{x}(0)
$$

Eq. (15) indicates that the action $S$ is only the function of the boundary values of state variables. Therefore, a symplectic pseudospectral method based on Eq. (15) that taking state variables at two ends of the interval as independent variables is proposed in the rest of this section.

The proposed method mainly consists of five steps. First, the whole time domain is divided into several sub-intervals. Then pseudospectral methods are applied to each sub-interval. After that, based on the principle of least action, a set of nonlinear algebraic equations can be obtained. Then, the boundary conditions are applied. Finally, the nonlinear algebraic equations are solved by Newton-Raphson iteration methods. Detailed descriptions are given as follow.

In the rest of this paper, unless otherwise stated, the superscript $(\cdot)^{(j)}$ refers to the value of the $j$-th sub-interval and $(\cdot)_{k}^{(j)}$ refers to the value at the $k$-th LGL point in the $j$-th sub-interval. While the subscript $(\cdot)_{j}$ refers to the value at the right end of the $j$-th sub-interval or the left end of the $(j+1)$-th sub-interval. And for problems with more than one degree of freedom, $(\cdot)_{[i]}$ refers to the value of the $i$-th degree of freedom.

\section{i. Discretizations of the time domain}

The whole time domain is divided into $P$ time intervals to conduct the numerical integration. For simplicity, one can divide the domain into $P$ regular intervals, which implies the length of each sub-interval equals to $\eta=t_{f} / P$. Thus the $j$-th sub-interval can be defined as $\left[t_{j-1}, t_{j}\right], j=1,2, \ldots, P$, where $t_{j-1}=(j-1) \eta$ and $t_{j}=j \eta$. Correspondingly, state variables and costate variables at $t_{j}$ are denoted as $\mathbf{x}_{j}$ and $\lambda_{j}$, respectively.

\section{ii. Applying pseudospectral methods to each sub-interval}

To apply pseudospectral methods, each sub-interval $t \in\left[t_{j-1}, t_{j}\right], j=1,2, \ldots, P$ should be transformed into the standard time interval $\tau^{(j)} \in[-1,1]$ by the following linear transformation

$$
\tau^{(j)}=\frac{2}{t_{j}-t_{j-1}}\left(t-\frac{t_{j}+t_{j-1}}{2}\right)
$$

Thus the action within the $j$-th sub-interval can be rewritten as 


$$
S^{(j)}=\frac{t_{j}-t_{j-1}}{2} \int_{-1}^{1}\left[\lambda^{\mathrm{T}} \frac{2}{t_{j}-t_{j-1}} \frac{d \mathbf{x}}{d \tau}-H(\mathbf{x}, \boldsymbol{\lambda}, \tau)\right] d \tau=\int_{-1}^{1}\left[\lambda^{\mathrm{T}} \frac{d \mathbf{x}}{d \tau}-\frac{t_{j}-t_{j-1}}{2} H(\mathbf{x}, \boldsymbol{\lambda}, \tau)\right] d \tau
$$

Let $L^{(j)}(\tau)$ be the $N^{(j)}$-order Legendre polynomial defined in the $j$-th sub-interval. Then, the LGL points $\tau_{k}^{(j)}, k=1,2, \ldots, N^{(j)}-1$ are roots of $\dot{L}^{(j)}(\tau)=0$, and together with $\tau_{0}^{(j)}=-1$ and $\tau_{N^{(j)}}^{(j)}=1$. Then the state variables and the costate variables within this interval can be approximated by values at LGL points as

$$
\begin{aligned}
& \mathbf{x}^{(j)}(\tau)=\sum_{l=0}^{N^{(j)}} \mathbf{x}\left(\tau_{l}^{(j)}\right) \phi_{l}^{(j)}(\tau) \\
& \lambda^{(j)}(\tau)=\sum_{l=0}^{N^{(j)}} \lambda\left(\tau_{l}^{(j)}\right) \phi_{l}^{(j)}(\tau)
\end{aligned}
$$

where

$$
\phi_{l}^{(j)}(\tau)=\frac{1}{N^{(j)}\left(N^{(j)}+1\right) L^{(j)}\left(\tau_{l}^{(j)}\right)} \cdot \frac{\left(\tau^{2}-1\right) \dot{L}^{(j)}(\tau)}{\tau-\tau_{l}^{(j)}}
$$

It is obvious that $\mathbf{x}\left(\tau_{0}^{(j)}\right)=\mathbf{x}_{j-1}, \mathbf{x}\left(\tau_{N^{(j)}}^{(j)}\right)=\mathbf{x}_{j}, \lambda\left(\tau_{0}^{(j)}\right)=\lambda_{j-1}$ and $\lambda\left(\tau_{N^{(j)}}^{(j)}\right)=\boldsymbol{\lambda}_{j}$. For simplicity, $\mathbf{x}\left(\tau_{k}^{(j)}\right)$ is denoted as $\mathbf{x}_{k}^{(j)}$, and $\lambda\left(\tau_{k}^{(j)}\right)$ is denoted as $\lambda_{k}^{(j)}$. In addition, $\overline{\mathbf{x}}^{(j)}$ and $\bar{\lambda}^{(j)}$ are defined as $\left[\left(\mathbf{x}_{1}^{(j)}\right)^{\mathrm{T}},\left(\mathbf{x}_{2}^{(j)}\right)^{\mathrm{T}}, \ldots,\left(\mathbf{x}_{N-1}^{(j)}\right)^{\mathrm{T}}\right]^{\mathrm{T}}$ and $\left[\left(\boldsymbol{\lambda}_{0}^{(j)}\right)^{\mathrm{T}},\left(\boldsymbol{\lambda}_{1}^{(j)}\right)^{\mathrm{T}}, \ldots,\left(\boldsymbol{\lambda}_{N}^{(j)}\right)^{\mathrm{T}}\right]^{\mathrm{T}}$, respectively.

Moreover, derivatives of state variables with respect to time at the LGL points are required to apply the Gauss-Lobatto integration rule to Eq. (17) to discretize the action. By differentiating Eq. (18), one obtains

$$
\frac{d}{d \tau} \mathbf{x}_{k}^{(j)}=\sum_{l=0}^{N^{(j)}} D_{k l}^{(j)} \mathbf{x}_{l}^{(j)}
$$

where

$$
\mathbf{D}^{(j)}=\left[D_{k l}^{(j)}\right]=\left\{\begin{array}{ccc}
-\frac{N^{(j)}\left(N^{(j)}+1\right)}{4} & , \quad k=l=0 \\
\frac{N^{(j)}\left(N^{(j)}+1\right)}{4} & , \quad k=l=N^{(j)} \\
\frac{L^{(j)}\left(\tau_{k}\right) \cdot \frac{1}{L^{(j)}\left(\tau_{l}\right)}}{\tau_{k}^{(j)}-\tau_{l}^{(j)}} & , \quad k \neq l \\
0 & \text { otherwise }
\end{array}\right.
$$


By substituting Eqs. (18), (19), (22) into Eq. (17) and applying the Gauss-Lobatto integration rule, the approximated action within the $j$-th sub-interval can be rewritten as

$$
S^{(j)}=\sum_{k=0}^{N^{(j)}} w_{k}^{(j)}\left[\left(\lambda_{k}^{(j)}\right)^{\mathrm{T}} \sum_{l=0}^{N^{(j)}} D_{k l}^{(j)} \mathbf{x}_{l}^{(j)}-\frac{t_{j}-t_{j-1}}{2} H\left(\mathbf{x}_{k}^{(j)}, \lambda_{k}^{(j)}, \theta_{k}^{(j)}\right)\right]
$$

where $w_{k}^{(j)}$ are the weight coefficients and $\theta_{k}^{(j)}$ are the time nodes corresponding to $\tau_{k}^{(j)}$

$$
\begin{gathered}
w_{k}^{(j)}=\frac{2}{N^{(j)}\left(N^{(j)}+1\right)} \cdot \frac{1}{\left[L^{(j)}\left(\tau_{k}^{(j)}\right)\right]^{2}} \\
\theta_{k}=\frac{t_{j}+t_{j-1}}{2}+\frac{t_{j}-t_{j-1}}{2} \tau_{k}^{(j)}
\end{gathered}
$$

\section{iii. Forming the nonlinear algebraic equations}

According to Eq. (15), if state variables at two ends of the $j$-th sub-interval, i.e., $\mathbf{x}_{j-1}$ and $\mathbf{x}_{j}$, are taken as independent variables, and Hamiltonian canonical equations in Eqs. (9) (10) are satisfied within the $j$-th sub-interval, the action within the $j$-th sub-interval must be the function of only $\mathbf{x}_{j-1}$ and $\mathbf{x}_{j}$. Hence, for satisfying the Hamiltonian equations, the action within the $j$-th sub-interval $S^{(j)}\left(\mathbf{x}_{j-1}, \mathbf{x}_{j}\right)$ can be expressed as

$$
S^{(j)}\left(\mathbf{x}_{j-1}, \mathbf{x}_{j}\right)=\operatorname{stat}_{\overline{\mathbf{x}}^{(j)}, \overline{\bar{x}}^{(j)}} S^{(j)}\left(\mathbf{x}_{j-1}, \overline{\mathbf{x}}^{(j)}, \mathbf{x}_{j}, \bar{\lambda}^{(j)}\right)
$$

where the symbol "stat" indicates that $\overline{\mathbf{x}}^{(j)}$ and $\bar{\lambda}^{(j)}$ are stationary points of $S^{(j)}$. Thus, stationary conditions in Eq. (26) can be expressed as

$$
\begin{aligned}
& \frac{\partial S^{(j)}\left(\mathbf{x}_{j-1}, \overline{\mathbf{x}}^{(j)}, \mathbf{x}_{j}, \bar{\lambda}^{(j)}\right)}{\partial \overline{\mathbf{x}}^{(j)}}=\mathbf{0} \\
& \frac{\partial S^{(j)}\left(\mathbf{x}_{j-1}, \overline{\mathbf{x}}^{(j)}, \mathbf{x}_{j}, \bar{\lambda}^{(j)}\right)}{\partial \bar{\lambda}^{(j)}}=\mathbf{0}
\end{aligned}
$$

To apply Eq. (15) to the whole time domain, the action in each sub-interval $S_{j}, j=1,2, \cdots, P$ should be summed up and the action within the whole time domain $S\left(\mathbf{x}_{0}, \mathbf{x}_{P}\right)$ can be obtained. Considering that $\mathbf{x}_{k}, k=1,2, \ldots P-1$ are stationary points of $S\left(\mathbf{x}_{0}, \mathbf{x}_{P}\right)$, one gets

$$
S\left(\mathbf{x}_{0}, \mathbf{x}_{P}\right)=\operatorname{stat}_{\mathbf{x}_{k}, k=1,2, \ldots, P}\left(\sum_{j=1}^{P} S^{(j)}\left(\mathbf{x}_{j}, \mathbf{x}_{j-1}\right)\right)
$$


Stationary conditions in Eq. (29) can be expressed as

$$
\frac{\partial S^{(j)}}{\mathbf{x}_{j}}+\frac{\partial S^{(j+1)}}{\mathbf{x}_{j}}=0, j=1,2, \ldots, P-1
$$

The costate variable at the two boundaries of the whole time domain can be obtained according to Eq. (15) as

$$
\lambda_{0}=-\frac{S\left(\mathbf{x}_{0}, \mathbf{x}_{P}\right)}{\partial \mathbf{x}_{0}}, \lambda_{P}=\frac{S\left(\mathbf{x}_{0}, \mathbf{x}_{P}\right)}{\partial \mathbf{x}_{P}}
$$

Thus, based on the principle of least action, the nonlinear optimal control problem has been transformed into a set of nonlinear algebraic equations consisting of Eqs. (27), (28), (30) and (31). In order to solve the nonlinear equations by Newton-Raphson iteration methods, the explicit expression of the Jacobian matrix should be derived in advance.

Considering the action within the $j$-th sub-interval, i.e., $S^{(j)}\left(\mathbf{x}_{j-1}, \mathbf{x}_{j}\right)$, differentiating it with respect to state and costate variables within the $j$-th sub-interval, one obtains

$$
\begin{gathered}
\left(\mathbf{f}_{0}^{x}\right)^{(j)}=-\lambda_{j-1} \\
\left(\mathbf{f}_{m}^{x}\right)^{(j)}=\mathbf{0}, m=1,2,3, \ldots, N^{(j)}-1 \\
\left(\mathbf{f}_{N}^{x}\right)^{(j)}=\lambda_{j} \\
\left(\mathbf{f}_{m}^{\lambda}\right)^{(j)}=\mathbf{0}, m=0,1,2, \ldots, N^{(j)}
\end{gathered}
$$

where $\left(\mathbf{f}_{m}^{x}\right)^{(j)}$ and $\left(\mathbf{f}_{m}^{\lambda}\right)^{(j)}$ are defined as

$$
\begin{gathered}
\left(\mathbf{f}_{m}^{x}\right)^{(j)}=\frac{\partial S^{(j)}}{\partial \mathbf{x}_{m}^{(j)}}=\sum_{k=0}^{N^{(j)}} w_{k}^{(j)} D_{k m}^{(j)} \lambda_{k}^{(j)}-w_{m}^{(j)} \frac{t_{j}-t_{j-1}}{2} \cdot \frac{\partial H\left(\mathbf{x}_{m}^{(j)}, \lambda_{m}^{(j)}, \theta_{m}^{(j)}\right)}{\partial \mathbf{x}_{m}^{(j)}} \\
\left(\mathbf{f}_{m}^{\lambda}\right)^{(j)}=\frac{\partial S^{(j)}}{\partial \lambda_{m}^{(j)}}=w_{m}^{(j)}\left(\sum_{k=0}^{N^{(j)}} D_{m k}^{(j)} \mathbf{x}_{k}^{(j)}-\frac{t_{j}-t_{j-1}}{2} \cdot \frac{\partial H\left(\mathbf{x}_{m}^{(j)}, \boldsymbol{\lambda}_{m}^{(j)}, \theta_{m}^{(j)}\right)}{\partial \lambda_{m}^{(j)}}\right)
\end{gathered}
$$

By differentiating Eqs. (36) and (37) with respect to $\mathbf{x}_{n}$ or $\lambda_{n}, n=0,1, \ldots, N^{(j)}$, one obtains

$$
\left(\mathbf{K}_{m n}^{x x}\right)^{(j)}=\frac{\left(\partial \mathbf{f}_{m}^{x}\right)^{(j)}}{\partial \mathbf{x}_{n}^{(j)}}=-\frac{t_{j}-t_{j-1}}{2} \cdot \frac{\partial^{2} H\left(\mathbf{x}_{m}^{(j)}, \lambda_{m}^{(j)}, \theta_{m}^{(j)}\right)}{\partial \mathbf{x}_{m}^{(j)} \partial \mathbf{x}_{n}^{(j)}} \delta_{m}^{n}
$$




$$
\begin{gathered}
\left(\mathbf{K}_{m n}^{x \lambda}\right)^{(j)}=\frac{\left(\partial \mathbf{f}_{m}^{x}\right)^{(j)}}{\partial \boldsymbol{\lambda}_{n}^{(j)}}=w_{n}^{(j)} D_{m n}^{(j)} \mathbf{I}-w_{m}^{(j)} \frac{t_{j}-t_{j-1}}{2} \cdot \frac{\partial^{2} H\left(\mathbf{x}_{m}^{(j)}, \boldsymbol{\lambda}_{m}^{(j)}, \theta_{m}^{(j)}\right)}{\partial \mathbf{x}_{m}^{(j)} \partial \lambda_{n}^{(j)}} \delta_{m}^{n} \\
\left(\mathbf{K}_{m n}^{\lambda x}\right)^{(j)}=\frac{\left(\partial \mathbf{f}_{m}^{\lambda}\right)^{(j)}}{\partial \mathbf{x}_{n}^{(j)}}=w_{m}^{(j)} D_{m n}^{(j)} \mathbf{I}-w_{m}^{(j)} \frac{t_{j}-t_{j-1}}{2} \cdot \frac{\partial^{2} H\left(\mathbf{x}_{m}^{(j)}, \boldsymbol{\lambda}_{m}^{(j)}, \theta_{m}^{(j)}\right)}{\partial \boldsymbol{\lambda}_{m}^{(j)} \partial \mathbf{x}_{n}^{(j)}} \delta_{m}^{n}=\left(\left(K_{n m}^{x \lambda}\right)^{(j)}\right)^{\mathrm{T}} \\
\left(\mathbf{K}_{m n}^{\lambda \lambda}\right)^{(j)}=\frac{\left(\partial \mathbf{f}_{m}^{\lambda}\right)^{(j)}}{\partial \lambda_{n}^{(j)}}=-w_{m}^{(j)} \frac{t_{j}-t_{j-1}}{2} \cdot \frac{\partial^{2} H\left(\mathbf{x}_{m}^{(j)}, \lambda_{m}^{(j)}, \theta_{m}^{(j)}\right)}{\partial \lambda_{m}^{(j)} \partial \lambda_{n}^{(j)}} \delta_{m}^{n}
\end{gathered}
$$

where $\mathbf{I}$ is the $d$-dimensional unit matrix and $\delta_{m}^{n}$ is the Kronecker delta. Till now, the components of the Jacobian matrix and the right-hand-side (RHS) vector generated by the $j$-th sub-interval, which are denoted as $\mathbf{K}^{(j)}$ and $\mathbf{f}^{(j)}$, respectively, can be expressed explicitly as

$$
\begin{gathered}
\mathbf{K}^{(j)}=\left[\begin{array}{llll}
\mathbf{K}_{11}^{(j)} & \mathbf{K}_{12}^{(j)} & \mathbf{K}_{13}^{(j)} & \mathbf{K}_{14}^{(j)} \\
\mathbf{K}_{21}^{(j)} & \mathbf{K}_{22}^{(j)} & \mathbf{K}_{23}^{(j)} & \mathbf{K}_{24}^{(j)} \\
\mathbf{K}_{31}^{(j)} & \mathbf{K}_{32}^{(j)} & \mathbf{K}_{33}^{(j)} & \mathbf{K}_{34}^{(j)} \\
\mathbf{K}_{41}^{(j)} & \mathbf{K}_{42}^{(j)} & \mathbf{K}_{43}^{(j)} & \mathbf{K}_{44}^{(j)}
\end{array}\right] \\
\mathbf{f}^{(j)}=\left[\left(\mathbf{F}_{1}^{(j)}\right)^{\mathrm{T}},\left(\mathbf{F}_{2}^{(j)}\right)^{\mathrm{T}},\left(\mathbf{F}_{3}^{(j)}\right)^{\mathrm{T}},\left(\mathbf{F}_{4}^{(j)}\right)^{\mathrm{T}}\right]^{\mathrm{T}}
\end{gathered}
$$

where detailed expressions of each sub-matrix in Eqs. (42) (43) are given in Appendix A.

Then with all variables arranged as

$$
\mathbf{v}=\left[\left(\mathbf{x}_{0}\right)^{\mathrm{T}},\left(\overline{\mathbf{x}}^{(1)}\right)^{\mathrm{T}},\left(\bar{\lambda}^{(1)}\right)^{\mathrm{T}} ;\left(\mathbf{x}_{1}\right)^{\mathrm{T}},\left(\overline{\mathbf{x}}^{(2)}\right)^{\mathrm{T}},\left(\bar{\lambda}^{(2)}\right)^{\mathrm{T}} ; \cdots ;\left(\mathbf{x}_{P-1}\right)^{\mathrm{T}},\left(\overline{\mathbf{x}}^{(P)}\right)^{\mathrm{T}},\left(\bar{\lambda}^{(P)}\right)^{\mathrm{T}} ;\left(\mathbf{x}_{P}\right)^{\mathrm{T}}\right]^{\mathrm{T}}
$$

One can obtain the explicit Jacobian matrix as follow

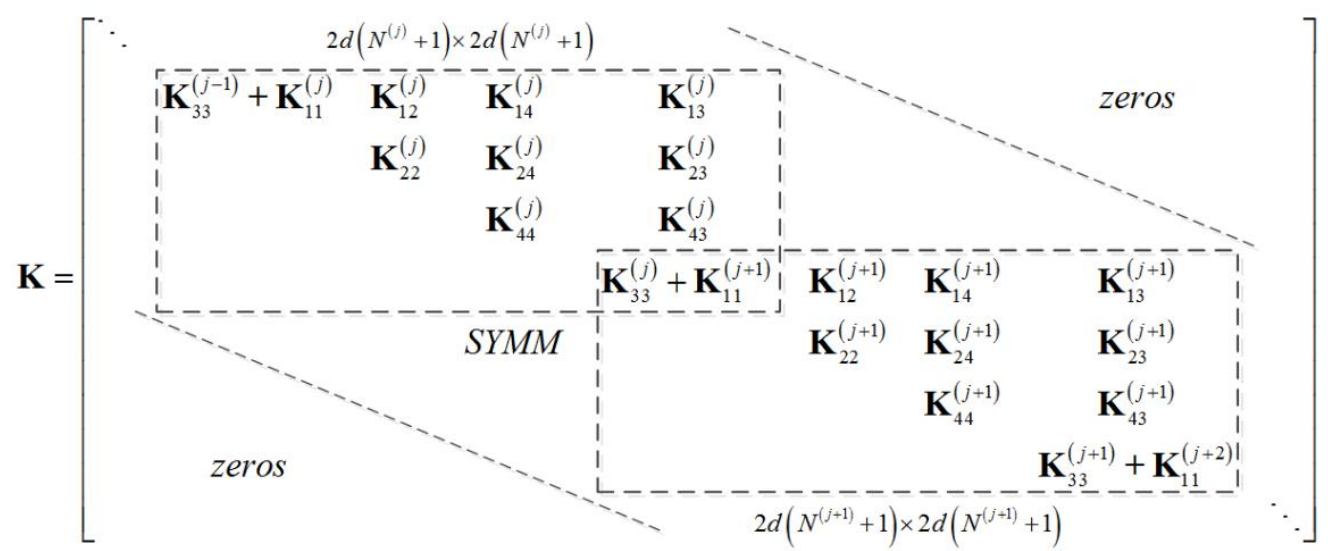

Correspondingly, the RHS of the nonlinear algebraic equations should be 


$$
\mathbf{F}=\left[\begin{array}{c}
{\left[\left(\mathbf{F}_{3}^{(j-1)}+\mathbf{F}_{1}^{(j)}\right)^{\mathrm{T}},\left(\mathbf{F}_{2}^{(j)}\right)^{\mathrm{T}},\left(\mathbf{F}_{4}^{(j)}\right)^{\mathrm{T}}\right]^{\mathrm{T}}} \\
{\left[\left(\mathbf{F}_{3}^{(j)}+\mathbf{F}_{1}^{(j+1)}\right)^{\mathrm{T}},\left(\mathbf{F}_{2}^{(j+1)}\right)^{\mathrm{T}},\left(\mathbf{F}_{4}^{(j+1)}\right)^{T}\right]^{\mathrm{T}}} \\
{\left[\left(\mathbf{F}_{3}^{(j+1)}+\mathbf{F}_{1}^{(j+2)}\right)^{\mathrm{T}},\left(\mathbf{F}_{2}^{(j+2)}\right)^{\mathrm{T}},\left(\mathbf{F}_{4}^{(j+2)}\right)^{T}\right]^{\mathrm{T}}} \\
\vdots
\end{array}\right]^{\mathrm{T}}
$$

Thus, the required Jacobian matrix $\mathbf{K}$ and the RHS vector $\mathbf{F}$ for solving the nonlinear equations are obtained. As can be seen in Eq. (45), together with expressions of each item given in Appendix A, $\mathbf{K}$ is a symmetric matrix. Moreover, $\mathbf{K}$ is sparse and with small band width. The value of the band width equals to $\max _{j=1,2, \ldots, P}\left[2 d\left(N^{(j)}+1\right)\right]$, which depends on the highest number of collocation points adopted in each sub-interval and the number of state variables. Therefore, the proposed method is highly efficient.

\section{iv. Treatments of the boundary conditions}

The Jacobian matrix $\mathbf{K}$ and the RHS vector $\mathbf{F}$ of the nonlinear equations should be modified according to the boundary conditions of the TPBVP. As it is said formerly, both fixed and free terminal states will be considered.

(1) fixed terminal states

In this case, boundary conditions are shown in Eq. (11). Since the values of state variables at the initial and terminal time nodes are given, Eq. (32) with $j=1$ and Eq. (34) with $j=P$ can be omitted. Correspondingly, the first and the last $d$ rows, together the first and the last $d$ columns of $\mathbf{K}$ should be deleted, and the first and the last $d$ rows of $\mathbf{F}$ are also deleted.

(2) free terminal states

For this case, boundary conditions are shown in Eq. (12). Similar to the fixed terminal case, Eq. (32) with $j=1$ can be omitted. Thus, the first $d$ rows and first $d$ columns of $\mathbf{K}$, and the first $d$ rows of F should be deleted. For simplicity, one assumes that the terminal performance index $h$ in Eq. (1) is a linear function of $\mathbf{x}\left(t_{f}\right)$ and then $\lambda_{f}$ become constant vectors that independent on $\mathbf{x}\left(t_{f}\right)$. Specially, $\lambda_{f}$ will be reduced to zero if the terminal performance index is not introduced into Eq. (1). 
Thus, $\lambda_{P}$ is removed from the list of unknown variables. Correspondingly, the penultimate $d$ rows and the penultimate $d$ columns of $\mathbf{K}$, together with the penultimate $d$ rows of $\mathbf{F}$ are deleted.

\section{v. The convergent criterion of nonlinear equations}

The convergent criterion needs to be proposed since the Newton-Raphson iteration method is adopted. Let $\mathbf{v}_{n}$ and $\mathbf{v}_{n+1}$ be the unknown variables in the $n$-th and the $(n+1)$-th iteration, then the criterion can be defined as

$$
\left\|\mathbf{v}_{n+1}-\mathbf{v}_{n}\right\| /\left\|\mathbf{v}_{n}\right\| \leq \varepsilon
$$

where $\varepsilon$ is a small quantity that denotes the acceptable error. After the converged unknown variables are obtained, the costate variables at the ends of each sub-interval should be modified by Eq. (31). Then the control input can be obtained by Eq. (6). Thus the nonlinear optimal control problem is solved.

\subsection{Proof of symplectic conserved approach}

The method proposed above can be proved as a symplectic conserved approach in this section. It is assumed that the numerical solution at two adjacent time node $t_{j-1}$ and $t_{j}$ in the discrete time domain are $\mathbf{v}_{j-1}$ and $\mathbf{v}_{j}$, respectively, where $\mathbf{v}_{j-1}=\left[\mathbf{x}_{j-1}^{\mathrm{T}}, \boldsymbol{\lambda}_{j-1}^{\mathrm{T}}\right]^{\mathrm{T}}$ and $\mathbf{v}_{j}=\left[\mathbf{x}_{j}^{\mathrm{T}}, \boldsymbol{\lambda}_{j}^{\mathrm{T}}\right]^{\mathrm{T}}$, subject to the function $\boldsymbol{\Psi}$ as

$$
\mathbf{v}_{j}=\Psi\left(\mathbf{v}_{j-1}\right)
$$

Whether the Jacobian matrix of the transfer function is a symplectic matrix determines whether the property of symplectic conservation applies to the algorithm. The Jacobian matrix of $\boldsymbol{\Psi}$ is expressed as

$$
\mathbf{Z}=\frac{\partial \mathbf{v}_{j}}{\partial \mathbf{v}_{j-1}}=\left[\begin{array}{ll}
\frac{\partial \mathbf{x}_{j}}{\partial \mathbf{x}_{j-1}} & \frac{\partial \mathbf{x}_{j}}{\partial \boldsymbol{\lambda}_{j-1}} \\
\frac{\partial \boldsymbol{\lambda}_{j}}{\partial \mathbf{x}_{j-1}} & \frac{\partial \boldsymbol{\lambda}_{j}}{\partial \boldsymbol{\lambda}_{j-1}}
\end{array}\right]
$$

The algorithm shown in Eq. (48) turns out to be a symplectic method when $\mathbf{Z}$ is a symplectic matrix, i.e., $\mathbf{Z} \mathbf{J} \mathbf{Z}^{\mathrm{T}}=\mathbf{J}$, where $\mathbf{J}$ is a unit symplectic matrix expressed as

$$
\mathbf{J}=\left[\begin{array}{cc}
0 & \mathbf{I} \\
-\mathbf{I} & 0
\end{array}\right]
$$

where $\mathbf{I}$ is a unit matrix shares the same size with $\frac{\partial \mathbf{x}_{j}}{\partial \mathbf{x}_{j-1}}$. 
By substituting Eq. (49) into Eq. (50), three sets of equations can be obtained as follow

$$
\begin{aligned}
& \left(\frac{\partial \mathbf{x}_{j}}{\partial \mathbf{x}_{j-1}}\right)^{\mathrm{T}} \frac{\partial \boldsymbol{\lambda}_{j}}{\partial \mathbf{x}_{j-1}}=\left[\left(\frac{\partial \mathbf{x}_{j}}{\partial \mathbf{x}_{j-1}}\right)^{\mathrm{T}} \frac{\partial \boldsymbol{\lambda}_{j}}{\partial \mathbf{x}_{j-1}}\right]^{\mathrm{T}} \\
& \left(\frac{\partial \mathbf{x}_{j}}{\partial \boldsymbol{\lambda}_{j-1}}\right)^{\mathrm{T}} \frac{\partial \boldsymbol{\lambda}_{j}}{\partial \boldsymbol{\lambda}_{j-1}}=\left[\left(\frac{\partial \mathbf{x}_{j}}{\partial \boldsymbol{\lambda}_{j-1}}\right)^{\mathrm{T}} \frac{\partial \boldsymbol{\lambda}_{j}}{\partial \boldsymbol{\lambda}_{j-1}}\right]^{\mathrm{T}} \\
& \left(\frac{\partial \mathbf{x}_{j}}{\partial \mathbf{x}_{j-1}}\right)^{\mathrm{T}} \frac{\partial \boldsymbol{\lambda}_{j}}{\partial \boldsymbol{\lambda}_{j-1}}-\left(\frac{\partial \boldsymbol{\lambda}_{j}}{\partial \mathbf{x}_{j-1}}\right)^{\mathrm{T}} \frac{\partial \mathbf{x}_{j}}{\partial \boldsymbol{\lambda}_{j-1}}=\mathbf{I}
\end{aligned}
$$

Theorem 1: For the nonlinear optimal control problem with given terminal time as shown in Eqs. (1) (2), the numerical method based on the least action principle express as Eq. (32) (35) is a symplectic conserved method, i.e., Eq.(51) (53) are satisfied.

Proof of Theorem 1: It is assumed that $\overline{\mathbf{x}}^{(j)}, \bar{\lambda}^{(j)}, \mathbf{x}_{j}$ and $\lambda_{j}$ are functions of $\mathbf{x}_{j-1}$ and $\lambda_{j-1}$ within the $j$-th sub-interval. By differentiating Eqs. (32) (35) with respect to $\mathbf{x}_{j-1}$, and according to the chain rules one obtains

$$
\begin{gathered}
\frac{\partial^{2} S^{(j)}}{\partial \mathbf{x}_{j-1} \partial \overline{\mathbf{x}}^{(j)}} \frac{\partial \overline{\mathbf{x}}^{(j)}}{\partial \mathbf{x}_{j-1}}+\frac{\partial^{2} S^{(j)}}{\partial \mathbf{x}_{j-1} \partial \bar{\lambda}^{(j)}} \frac{\partial \bar{\lambda}^{(j)}}{\partial \mathbf{x}_{j-1}}+\frac{\partial^{2} S^{(j)}}{\partial \mathbf{x}_{j-1} \partial \mathbf{x}_{j}} \frac{\partial \mathbf{x}_{j}}{\partial \mathbf{x}_{j-1}}+\frac{\partial^{2} S^{(j)}}{\partial \mathbf{x}_{j-1} \partial \mathbf{x}_{j-1}}=\mathbf{0} \\
\frac{\partial^{2} S^{(j)}}{\partial \overline{\mathbf{x}}^{(j)} \partial \overline{\mathbf{x}}^{(j)}} \frac{\partial \overline{\mathbf{x}}^{(j)}}{\partial \mathbf{x}_{j-1}}+\frac{\partial^{2} S^{(j)}}{\partial \overline{\mathbf{x}}^{(j)} \partial \bar{\lambda}^{(j)}} \frac{\partial \bar{\lambda}^{(j)}}{\partial \mathbf{x}_{j-1}}+\frac{\partial^{2} S^{(j)}}{\partial \overline{\mathbf{x}}^{(j)} \partial \mathbf{x}_{j}} \frac{\partial \mathbf{x}_{j}}{\partial \mathbf{x}_{j-1}}+\frac{\partial^{2} S^{(j)}}{\partial \overline{\mathbf{x}}^{(j)} \partial \mathbf{x}_{j-1}}=\mathbf{0} \\
\frac{\partial^{2} S^{(j)}}{\partial \mathbf{x}_{j} \partial \overline{\mathbf{x}}^{(j)}} \frac{\partial \overline{\mathbf{x}}^{(j)}}{\partial \mathbf{x}_{j-1}}+\frac{\partial^{2} S^{(j)}}{\partial \mathbf{x}_{j} \partial \bar{\lambda}^{(j)}} \frac{\partial \bar{\lambda}^{(j)}}{\partial \mathbf{x}_{j-1}}+\frac{\partial^{2} S^{(j)}}{\partial \mathbf{x}_{j} \partial \mathbf{x}_{j}} \frac{\partial \mathbf{x}_{j}}{\partial \mathbf{x}_{j-1}}+\frac{\partial^{2} S^{(j)}}{\partial \mathbf{x}_{j} \partial \mathbf{x}_{j-1}}=\frac{\partial \boldsymbol{\lambda}_{j}}{\partial \mathbf{x}_{j-1}} \\
\frac{\partial^{2} S^{(j)}}{\partial \bar{\lambda}^{(j)} \partial \overline{\mathbf{x}}^{(j)}} \frac{\partial \overline{\mathbf{x}}^{(j)}}{\partial \mathbf{x}_{j-1}}+\frac{\partial^{2} S^{(j)}}{\partial \bar{\lambda}^{(j)} \partial \bar{\lambda}^{(j)}} \frac{\partial \bar{\lambda}^{(j)}}{\partial \mathbf{x}_{j-1}}+\frac{\partial^{2} S^{(j)}}{\partial \bar{\lambda}^{(j)} \partial \mathbf{x}_{j}} \frac{\partial \mathbf{x}_{j}}{\partial \mathbf{x}_{j-1}}+\frac{\partial^{2} S^{(j)}}{\partial \bar{\lambda}^{(j)} \partial \mathbf{x}_{j-1}}=\mathbf{0}
\end{gathered}
$$

By simplifying Eqs. (54) (57), one obtains

$$
\begin{aligned}
& \boldsymbol{\Gamma}_{1} \mathbf{X}+\boldsymbol{\Gamma}_{2}{ }^{\mathrm{T}}=\left[\begin{array}{lll}
\mathbf{0} & \mathbf{0} & \left(\frac{\partial \boldsymbol{\lambda}_{j}}{\partial \mathbf{x}_{j-1}}\right)^{\mathrm{T}}
\end{array}\right]^{\mathrm{T}} \\
& \boldsymbol{\Gamma}_{2}{ }^{\mathrm{T}} \mathbf{X}=-\frac{\partial^{2} S^{(j)}}{\partial \mathbf{x}_{j-1} \partial \mathbf{x}_{j-1}}
\end{aligned}
$$

where 


$$
\begin{gathered}
\boldsymbol{\Gamma}_{1}=\left[\begin{array}{lll}
\frac{\partial^{2} S^{(j)}}{\partial \overline{\mathbf{x}}^{(j)} \partial \overline{\mathbf{x}}^{(j)}} & \frac{\partial^{2} S^{(j)}}{\partial \overline{\mathbf{x}}^{(j)} \partial \overline{\boldsymbol{\lambda}}^{(j)}} & \frac{\partial^{2} S^{(j)}}{\partial \overline{\mathbf{x}}^{(j)} \partial \mathbf{x}_{j}} \\
\frac{\partial^{2} S^{(j)}}{\partial \overline{\boldsymbol{\lambda}}^{(j)} \partial \overline{\mathbf{x}}^{(j)}} & \frac{\partial^{2} S^{(j)}}{\partial \overline{\boldsymbol{\lambda}}^{(j)} \partial \overline{\boldsymbol{\lambda}}^{(j)}} & \frac{\partial^{2} S^{(j)}}{\partial \overline{\boldsymbol{\lambda}}^{(j)} \partial \mathbf{x}_{j}} \\
\frac{\partial^{2} S^{(j)}}{\partial \mathbf{x}_{j} \partial \overline{\mathbf{x}}^{(j)}} & \frac{\partial^{2} S^{(j)}}{\partial \mathbf{x}_{j} \partial \overline{\boldsymbol{\lambda}}^{(j)}} & \frac{\partial^{2} S^{(j)}}{\partial \mathbf{x}_{j} \partial \mathbf{x}_{j}}
\end{array}\right] \\
\boldsymbol{\Gamma}_{2}=\left[\begin{array}{lll}
\frac{\partial^{2} S^{(j)}}{\partial \overline{\mathbf{x}}^{(j)} \partial \mathbf{x}_{j-1}} & \frac{\partial^{2} S^{(j)}}{\partial \overline{\boldsymbol{\lambda}}^{(j)} \partial \mathbf{x}_{j-1}} & \frac{\partial^{2} S^{(j)}}{\partial \mathbf{x}_{j} \partial \mathbf{x}_{j-1}}
\end{array}\right] \\
\mathbf{X}=\left[\begin{array}{lll}
\left(\frac{\partial \overline{\mathbf{x}}^{(j)}}{\partial \mathbf{x}_{j-1}}\right)^{\mathrm{T}} & \left(\frac{\partial \overline{\boldsymbol{\lambda}}^{(j)}}{\partial \mathbf{x}_{j-1}}\right)^{\mathrm{T}} & \left(\frac{\partial \mathbf{x}_{j}}{\partial \mathbf{x}_{j-1}}\right)^{\mathrm{T}}
\end{array}\right]^{\mathrm{T}}
\end{gathered}
$$

Similarly, by differentiating Eqs. (32) (35) with respect to $\lambda_{j-1}$, one obtains

$$
\begin{gathered}
\frac{\partial^{2} S^{(j)}}{\partial \mathbf{x}_{j-1} \partial \overline{\mathbf{x}}^{(j)}} \frac{\partial \overline{\mathbf{x}}^{(j)}}{\partial \boldsymbol{\lambda}_{j-1}}+\frac{\partial^{2} S^{(j)}}{\partial \mathbf{x}_{j-1} \partial \overline{\boldsymbol{\lambda}}^{(j)}} \frac{\partial \overline{\mathbf{x}}^{(j)}}{\partial \boldsymbol{\lambda}_{j-1}}+\frac{\partial^{2} S^{(j)}}{\partial \mathbf{x}_{j-1} \partial \mathbf{x}_{j}} \frac{\partial \overline{\mathbf{x}}^{(j)}}{\partial \boldsymbol{\lambda}_{j-1}}=-\mathbf{I} \\
\frac{\partial^{2} S^{(j)}}{\partial \overline{\mathbf{x}}^{(j)} \partial \overline{\mathbf{x}}^{(j)}} \frac{\partial \overline{\mathbf{x}}^{(j)}}{\partial \boldsymbol{\lambda}_{j-1}}+\frac{\partial^{2} S^{(j)}}{\partial \overline{\mathbf{x}}^{(j)} \partial \overline{\boldsymbol{\lambda}}^{(j)}} \frac{\partial \overline{\boldsymbol{\lambda}}^{(j)}}{\partial \boldsymbol{\lambda}_{j-1}}+\frac{\partial^{2} S^{(j)}}{\partial \overline{\mathbf{x}}^{(j)} \partial \mathbf{x}_{j}} \frac{\partial \mathbf{x}_{j}}{\partial \boldsymbol{\lambda}_{j-1}}=\mathbf{0} \\
\frac{\partial^{2} S^{(j)}}{\partial \mathbf{x}_{j} \partial \overline{\mathbf{x}}^{(j)}} \frac{\partial \overline{\mathbf{x}}^{(j)}}{\partial \boldsymbol{\lambda}_{j-1}}+\frac{\partial^{2} S^{(j)}}{\partial \mathbf{x}_{j} \partial \overline{\boldsymbol{\lambda}}^{(j)}} \frac{\partial \overline{\boldsymbol{\lambda}}^{(j)}}{\partial \boldsymbol{\lambda}_{j-1}}+\frac{\partial^{2} S^{(j)}}{\partial \mathbf{x}_{j} \partial \mathbf{x}_{j}} \frac{\partial \mathbf{x}_{j}}{\partial \boldsymbol{\lambda}_{j-1}}=\frac{\partial \boldsymbol{\lambda}_{j}}{\partial \boldsymbol{\lambda}_{j-1}} \\
\frac{\partial^{2} S^{(j)}}{\partial \overline{\boldsymbol{\lambda}}^{(j)} \partial \overline{\mathbf{x}}^{(j)}} \frac{\partial \overline{\mathbf{x}}^{(j)}}{\partial \boldsymbol{\lambda}_{j-1}}+\frac{\partial^{2} S^{(j)}}{\partial \overline{\boldsymbol{\lambda}}^{(j)} \partial \overline{\boldsymbol{\lambda}}^{(j)}} \frac{\partial \overline{\boldsymbol{\lambda}}^{(j)}}{\partial \boldsymbol{\lambda}_{j-1}}+\frac{\partial^{2} S^{(j)}}{\partial \overline{\boldsymbol{\lambda}}^{(j)} \partial \mathbf{x}_{j}} \frac{\partial \mathbf{x}_{j}}{\partial \boldsymbol{\lambda}_{j-1}}=\mathbf{0}
\end{gathered}
$$

Then, by reducing Eqs. (63) (66), one obtains

$$
\begin{gathered}
\boldsymbol{\Gamma}_{1} \mathbf{Y}=\left[\begin{array}{lll}
\mathbf{0} & \mathbf{0} & \left(\frac{\partial \boldsymbol{\lambda}_{j}}{\partial \boldsymbol{\lambda}_{j-1}}\right)^{\mathrm{T}}
\end{array}\right]^{\mathrm{T}} \\
\boldsymbol{\Gamma}_{2}{ }^{\mathrm{T}} \mathbf{Y}=-\mathbf{I}
\end{gathered}
$$

where

$$
\mathbf{Y}=\left[\left(\frac{\partial \overline{\mathbf{x}}^{(j)}}{\partial \boldsymbol{\lambda}_{j-1}}\right)^{\mathrm{T}}\left(\frac{\partial \overline{\boldsymbol{\lambda}}^{(j)}}{\partial \boldsymbol{\lambda}_{j-1}}\right)^{\mathrm{T}}\left(\frac{\partial \mathbf{x}_{j}}{\partial \boldsymbol{\lambda}_{j-1}}\right)^{\mathrm{T}}\right]^{\mathrm{T}}
$$

Left multiplying Eq. (68) by $\mathbf{X}^{\mathrm{T}}$, one obtains 


$$
\mathbf{X}^{\mathrm{T}} \boldsymbol{\Gamma}_{1} \mathbf{X}+\mathbf{X}^{\mathrm{T}} \boldsymbol{\Gamma}_{2}^{\mathrm{T}}=\left(\frac{\partial \mathbf{x}_{j}}{\partial \mathbf{x}_{j-1}}\right)^{\mathrm{T}}\left(\frac{\partial \boldsymbol{\lambda}_{j}}{\partial \mathbf{x}_{j-1}}\right)
$$

Substituting Eq. (59) into Eq. (70), one obtains

$$
\mathbf{X}^{\mathrm{T}} \boldsymbol{\Gamma}_{1} \mathbf{X}-\frac{\partial^{2} S^{(j)}}{\partial \mathbf{x}_{j-1} \partial \mathbf{x}_{j-1}}=\left(\frac{\partial \mathbf{x}_{j}}{\partial \mathbf{x}_{j-1}}\right)^{\mathrm{T}}\left(\frac{\partial \boldsymbol{\lambda}_{j}}{\partial \mathbf{x}_{j-1}}\right)
$$

Then Eq. (52) is satisfied since matrix $\left(\frac{\partial \mathbf{x}_{j}}{\partial \mathbf{x}_{j-1}}\right)^{\mathrm{T}}\left(\frac{\partial \boldsymbol{\lambda}_{j}}{\partial \mathbf{x}_{j-1}}\right)$ is a symmetric matrix and it also can be seen in Eq. (71).

Left multiplying the Eq. (67) by $\mathbf{Y}^{\mathrm{T}}$, one obtains

$$
\mathbf{Y}^{\mathrm{T}} \boldsymbol{\Gamma}_{1} \mathbf{Y}=\left(\frac{\partial \mathbf{x}_{j}}{\partial \boldsymbol{\lambda}_{j-1}}\right)^{\mathrm{T}}\left(\frac{\partial \boldsymbol{\lambda}_{j}}{\partial \boldsymbol{\lambda}_{j-1}}\right)
$$

Then Eq. (52) is satisfied automatically as can be seen in Eq. (72).

Finally, left multiplying the Eq. (67) by $\mathbf{X}^{\mathrm{T}}$, one obtains

$$
\mathbf{X}^{\mathrm{T}} \boldsymbol{\Gamma}_{1} \mathbf{Y}=\left(\frac{\partial \mathbf{x}_{j}}{\partial \mathbf{x}_{j-1}}\right)^{\mathrm{T}}\left(\frac{\partial \boldsymbol{\lambda}_{j}}{\partial \boldsymbol{\lambda}_{j-1}}\right)
$$

Right multiplying the transposition of Eq. (58) by $\mathbf{Y}$, one obtains

$$
\mathbf{X}^{\mathrm{T}} \boldsymbol{\Gamma}_{1} \mathbf{Y}+\boldsymbol{\Gamma}_{2} \mathbf{Y}=\left(\frac{\partial \boldsymbol{\lambda}_{j}}{\partial \mathbf{x}_{j-1}}\right)^{\mathrm{T}}\left(\frac{\partial \mathbf{x}_{j}}{\partial \boldsymbol{\lambda}_{j-1}}\right)
$$

Substituting Eq. (73) into Eq. (74), $\boldsymbol{\Gamma}_{2} \mathbf{Y}$ can be expressed as

$$
\boldsymbol{\Gamma}_{2} \mathbf{Y}=\left(\frac{\partial \boldsymbol{\lambda}_{j}}{\partial \mathbf{x}_{j-1}}\right)^{\mathrm{T}}\left(\frac{\partial \mathbf{x}_{j}}{\partial \boldsymbol{\lambda}_{j-1}}\right)-\left(\frac{\partial \mathbf{x}_{j}}{\partial \mathbf{x}_{j-1}}\right)^{\mathrm{T}}\left(\frac{\partial \boldsymbol{\lambda}_{j}}{\partial \boldsymbol{\lambda}_{j-1}}\right)
$$

Then introducing Eq. (68) into Eq. (75), Eq. (53) is satisfied. Thus, Theorem 1 is proved up since Eqs. (51) (53) are satisfied.

\section{The adaptive implementation of symplectic pseudospectral methods}

The adaptive mesh refinement method is described in this section. The error estimation method based on the residual error of the dynamic constraints in each sub-interval is given first. Then the criterion of whether the sub-interval should be modified is described. After that, two methods of modifying the sub-interval, i.e., dividing the sub-interval into more sub-intervals ( $h$ methods) and 
increasing the degree of the approximation polynomial ( $p$ methods), are discussed in detail. Finally the flow chart diagram of the algorithm proposed is presented.

\subsection{Error estimations in each sub-interval}

Since the dynamic constrains shown in Eq. (2) is given, the residual error of the dynamic constraints at the $k$-th LGL point of the $j$-th sub-interval is defined as

$$
e_{k}^{(j)}=\left\|\dot{\mathbf{x}}_{k}^{(j)}-\frac{t_{j}-t_{j-1}}{2} \mathbf{f}\left(\mathbf{x}_{k}^{(j)}, \lambda_{k}^{(j)}, \theta_{k}^{(j)}\right)\right\|_{\infty}=\left\|\sum_{l=0}^{N^{(j)}} D_{k l}^{(j)} \mathbf{x}_{l}^{(j)}-\frac{t_{j}-t_{j-1}}{2} \mathbf{f}\left(\mathbf{x}_{k}^{(j)}, \lambda_{k}^{(j)}, \theta_{k}^{(j)}\right)\right\|_{\infty}
$$

and the maximum residual error within the $j$-th sub-interval is defined as

$$
e_{\max }^{(j)}=\max _{k=0,1, \ldots, N^{(j)}}\left(e_{k}^{(j)}\right)
$$

\subsection{The refining criterion}

Then the mesh within $j$-th sub-interval should be refined by either $h$ methods or $p$ methods when $e_{\max }^{(j)}$ exceeds $\mu$, where $\mu$ is the mesh refinement accuracy tolerance. It is obvious that the final solution is obtained when the max residual error meets the demand of accuracy in every sub-interval. Then a criterion based on the relative curvature of the state variables is adopted to decide which method should be taken to refine the mesh.

Let $k_{q,[m]}^{(j)}$ be the curvature of the $m$-th degree of freedom of the state at the $q$-th LGL point of the $j$-th sub-interval, where $q=1,2, \cdots, N^{(j)}$ and $m=1,2, \cdots, d$. The maximum curvature of all $d$ degrees of freedom is required as a reference to conduct the adaptive procedure and is defined as

$$
k_{q}^{(j)}=\max _{m=1,2, \ldots, d}\left(k_{q,[m]}^{(j)}\right)=\max _{m=1,2, \cdots, d}\left|\frac{\ddot{\mathbf{x}}_{q,[m]}^{(j)}}{\left[1+\left(\dot{\mathbf{x}}_{q,[m]}^{(j)}\right)^{2}\right]^{3 / 2}}\right|
$$

As can be seen from Eq. (78), the second order differential matrix of state variables $\mathbf{C}^{(j)}$ is required to calculate $k_{q}^{(j)}$ and given by [42-43]

$$
\mathbf{C}^{(j)}=\mathbf{D}^{(j)} \mathbf{D}^{(j)}
$$

Substituting Eq. (79) into Eq. (78), one gets 


$$
k_{q}^{(j)}=\max _{m=1,2, \cdots, d}\left|\frac{\sum_{l=0}^{N^{(j)}} C_{q l}^{(j)} \mathbf{x}_{l,[m]}^{(j)}}{\left[1+\left(\sum_{l=0}^{N^{(j)}} D_{q l}^{(j)} \mathbf{x}_{l, l[m]}^{(j)}\right)^{2}\right]^{3 / 2}}\right|
$$

And $k_{\max }^{(j)}$ and $k_{a v e}^{(j)}$ are defined as

$$
\begin{gathered}
k_{\max }^{(j)}=\max _{q=0,1, \ldots, N^{(j)}}\left(k_{q}^{(j)}\right) \\
k_{\text {ave }}^{(j)}=\frac{\sum_{q=0}^{N^{(j)}} k_{q}^{(j)}}{N^{(j)}+1}
\end{gathered}
$$

Then the relative curvature $r^{(j)}$ is introduced, which is defined as

$$
r^{(j)}=k_{\max }^{(j)} / k_{\text {ave }}^{(j)}
$$

A threshold $R$ is set. Two conditions, i.e. $r^{(j)} \leq R$ and $r^{(j)}>R$, are discussed below.

(1) Case of $r^{(j)} \leq R$

In this case, the trajectory of state variables within the $j$-th sub-interval is considered to be relatively smooth. Then the degree of the interpolation function in this sub-interval increases to $N_{\text {new }}^{(j)}$, which implies more interpolation points will be used, where

$$
N_{\text {new }}^{(j)}=N_{\text {old }}^{(j)}+\text { ceil }\left[\lg \left(e_{\max }^{(j)} / \mu\right)\right]+A
$$

where ceil $(\square)$ is the function that rounds the number upwards and $A$ is a positive integer. As can be seen from Eq. (84), if $\mu$ and $A$ are given, the larger the residual error of the sub-interval is, the more collocation points will be increased in the $j$-th sub-interval. This is a $p$-method in essence as the more collocation points are adopted, the more accurate solution one may obtains.

(2) Case of $r^{(j)}>R$

As for this case, in contrast to the former case, the trajectory of state variables within the $j$-th sub-interval is considered to be so oscillating that the sub-interval should be refined. Then this sub-interval should be divided into $M^{(j)}$ smaller equidistant sub-intervals, where

$$
M^{(j)}=\operatorname{ceil}\left[B \cdot \lg \left(e_{\max }^{(j)} / \mu\right)\right]
$$


where $B$ is a positive real number no smaller than 1 . As can be seen in Eq. (85), the $j$-th sub-interval may be divided into more small time intervals as the residual error gets larger when $\mu$ and $B$ are fixed. A refined mesh with more sub-intervals can be obtained by this procedure, which leads it to be an $h$-method.

\subsection{Design of the $h p$ adaptive algorithm}

Step (1): Set global parameters, such as $\varepsilon, \mu, R, A, B$ and iterMax.

Step (2): Let $P$ be the number of sub-intervals. Give an initial mesh which consists of $P$ sub-intervals, i.e., $Q^{(j)}=\left[t_{j-1}, t_{j}\right], j=1,2, \ldots, P$, with an $N^{(j)}$-degree interpolation function used in each sub-interval.

Step (3): Solve the optimal control problem by the method proposed in section 3 with current mesh.

Step (4): Terminate the program if the maximum residual error defined in Eq. (77) meets the demand of accuracy in every $P$ sub-intervals or the iterations meet the given upper limit, i.e., iterMax. Otherwise, modify the mesh as discussed in section 4.2 and go back to step (3).

Finally, a flow chart of the above $h p$ adaptive algorithm is given as follow, where three mesh parameters, i.e., $P, Q^{(j)}$ (or $Q Q^{(j)}$ ) and $N^{(j)}$ (or $N N^{(j)}$ ) represent the number of sub-intervals, the location of the $j$-th sub-interval and the degree of approximation polynomial used in the $j$-th sub-interval, respectively. 


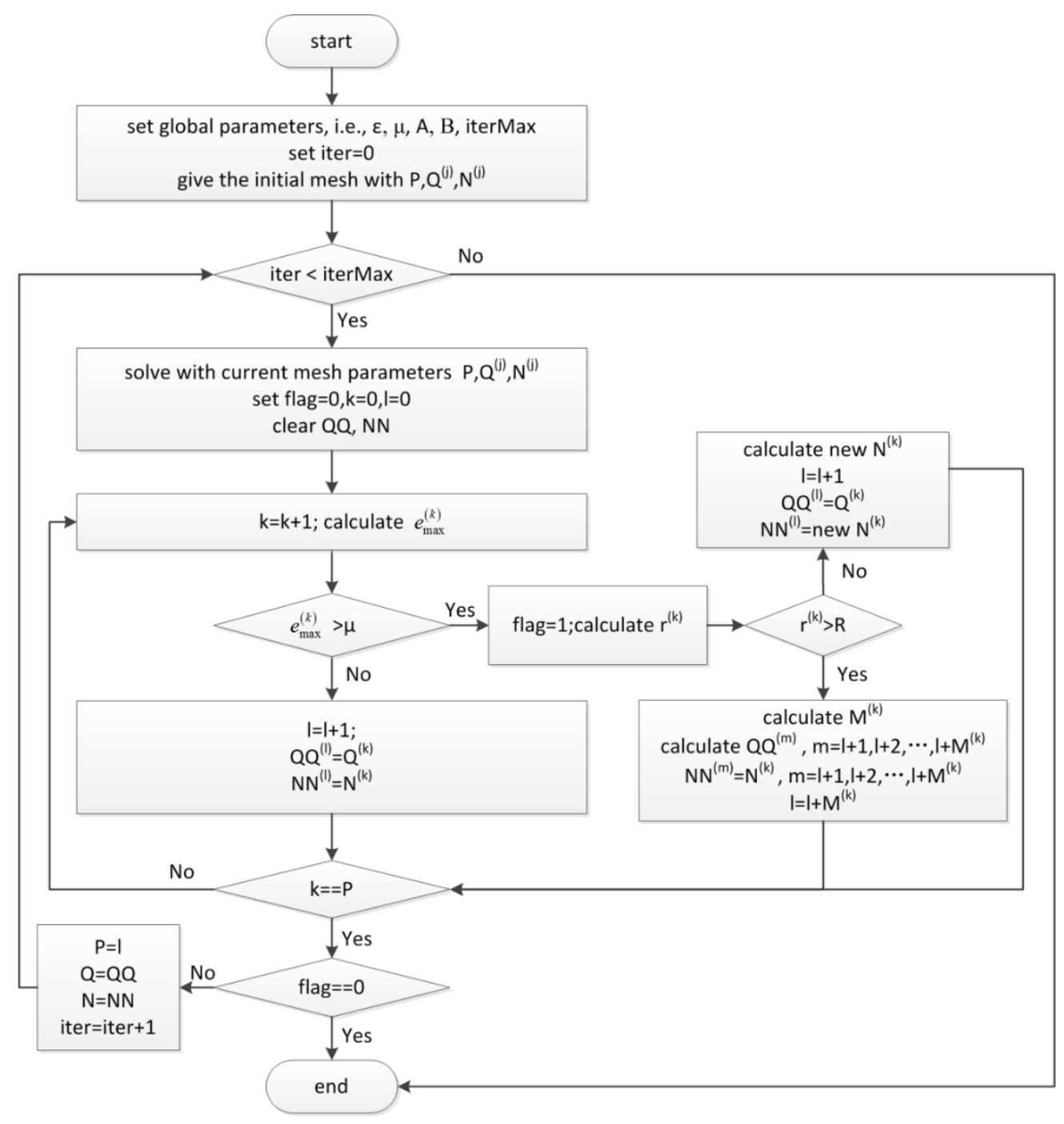

Fig. 1 Flow chart diagram of the $h p$ adaptive symplectic pseudospectral method

\section{Numerical examples}

In this section the symplectic pseudospectral method described in Section 3 and the adaptive method given in Section 4 are applied to five examples form open literatures. The first example is an optimal control problem with fixed terminal states described in reference [23], where the rate of convergence of the proposed method is discussed. In the second example, a Van der Pol problem with a free terminal end given in reference [21] is discussed to validate the effectiveness of the proposed method for optimal control problems with free terminal states. Furthermore, the influence of the distribution of sub-intervals is provided in the second example. Then a hyper-sensitive optimal control problem described in reference [27] is given as the third example, where the adaptive method is applied to show it leads to a reasonable mesh. Then, two astrodynamics problems are given to test the 
effectiveness of the proposed algorithms for solving engineering problems. The first example is a complex problem with respect to formation reconfiguration of satellites described in reference [35]. And the second example is to simulating the deployment procedure of a two-body tethered satellite system given in reference [44].

The convergent criteria given in Eq. (47) of the first three examples are set to be 1.0e-15 and the last two to be 1.0e-10. And the initial guesses of unknown state and costate variables at each discrete time nodes in Newton-Raphson iterations are all set to be the values of state variables at the initial time. Finally, it is noted that all computations are performed in MATLAB (R2014a) on an Intel ${ }^{\circledR}$ Core $^{\mathrm{TM}}$ i7-4710MQ machine with a $2.50 \mathrm{GHz}$ processor and $4 \mathrm{~GB}$ of RAM.

Example 1: Consider a one-dimensional optimal control problem with fixed terminal state. One aims to minimize the cost functional

$$
J=\frac{1}{2} \int_{0}^{5}\left(x+u^{2}\right) d t
$$

subject to the dynamic constraint

$$
\dot{x}=2 x+2 u \sqrt{x}
$$

and boundary conditions

$$
x(0)=2, x(5)=1
$$

It is noted that the analytical solution for this problem is available in [23]. The proposed method and the symplectic method proposed in reference [32] are both adopted respectively to solve the problem. And the efficiencies of the two methods are compared with respect to various performance indices. In order to analyze the influence of the number of sub-intervals and the degree of the interpolation function on the decision, the whole time domain is divided into equidistant sub-intervals with the interpolation functions of the same degree in all sub-intervals in this example. Let $N$ and $m$ be the number of sub-intervals and the degree of interpolation function applied to state variables in each sub-interval, respectively, in both the proposed method and the symplecitc method. For the sake of simplicity, we only present the results of the second type of the symplectic method [32] where the degrees of the interpolation function of state and costate variables are equal, which is similar to the proposed method. Thus, there are the same amounts of unknown variables in the proposed method and the symplectic method with the same $N$ and the same $m$, which makes the comparison much more 
convincible. The results are given in Table 1, where precision refers to the relative error of state variables calculated by value at ends of sub-intervals.

Table 1 Effectiveness of two methods with various $N$ and $m$

\begin{tabular}{cccccccc}
\hline \hline \multirow{2}{*}{$N$} & $m$ & \multicolumn{2}{c}{ CPU times/s } & \multicolumn{2}{c}{ Precision } & \multicolumn{2}{c}{ Iterations } \\
& & PM & SM & PM & SM & PM & SM \\
\hline \multirow{4}{*}{4} & 4 & 0.029 & 0.787 & $8.335 \mathrm{e}-5$ & $2.911 \mathrm{e}-3$ & 10 & 45 \\
& 5 & 0.042 & 0.680 & $2.039 \mathrm{e}-6$ & $1.239 \mathrm{e}-4$ & 13 & 35 \\
& 8 & 0.059 & 0.716 & $2.522 \mathrm{e}-11$ & $4.257 \mathrm{e}-10$ & 12 & 29 \\
& 11 & 0.074 & 0.706 & $7.733 \mathrm{e}-16$ & $8.489 \mathrm{e}-15$ & 12 & 17 \\
8 & 4 & 0.054 & 0.813 & $7.017 \mathrm{e}-7$ & $1.639 \mathrm{e}-4$ & 12 & 23 \\
& 5 & 0.073 & 0.979 & $1.298 \mathrm{e}-8$ & $1.274 \mathrm{e}-6$ & 12 & 25 \\
& 8 & 0.123 & 1.116 & $7.409 \mathrm{e}-16$ & $1.209 \mathrm{e}-13$ & 11 & 17 \\
& 11 & 0.199 & 2.420 & $2.902 \mathrm{e}-16$ & $2.910 \mathrm{e}-15$ & 11 & 31 \\
& 4 & 0.109 & 1.693 & $1.731 \mathrm{e}-9$ & $2.271 \mathrm{e}-6$ & 11 & 21 \\
& 5 & 0.156 & 1.894 & $2.601 \mathrm{e}-11$ & $6.794 \mathrm{e}-9$ & 11 & 21 \\
& 8 & 0.282 & 2.921 & $3.68 \mathrm{ee}-16$ & $3.597 \mathrm{e}-15$ & 11 & 27 \\
& 11 & 0.470 & 5.669 & $3.998 \mathrm{e}-16$ & $2.738 \mathrm{e}-15$ & 12 & 37 \\
& 4 & 0.235 & 4.720 & $4.569 \mathrm{e}-12$ & $4.192 \mathrm{e}-8$ & 11 & 29 \\
& 5 & 0.333 & 4.993 & $3.344 \mathrm{e}-14$ & $3.153 \mathrm{e}-11$ & 11 & 27 \\
& 8 & 0.830 & 4.047 & $4.451 \mathrm{e}-16$ & $4.428 \mathrm{e}-16$ & 15 & 29 \\
\hline \hline
\end{tabular}

Remark : PM: Proposed method SM: the second type of the symplectic method in reference [32] 

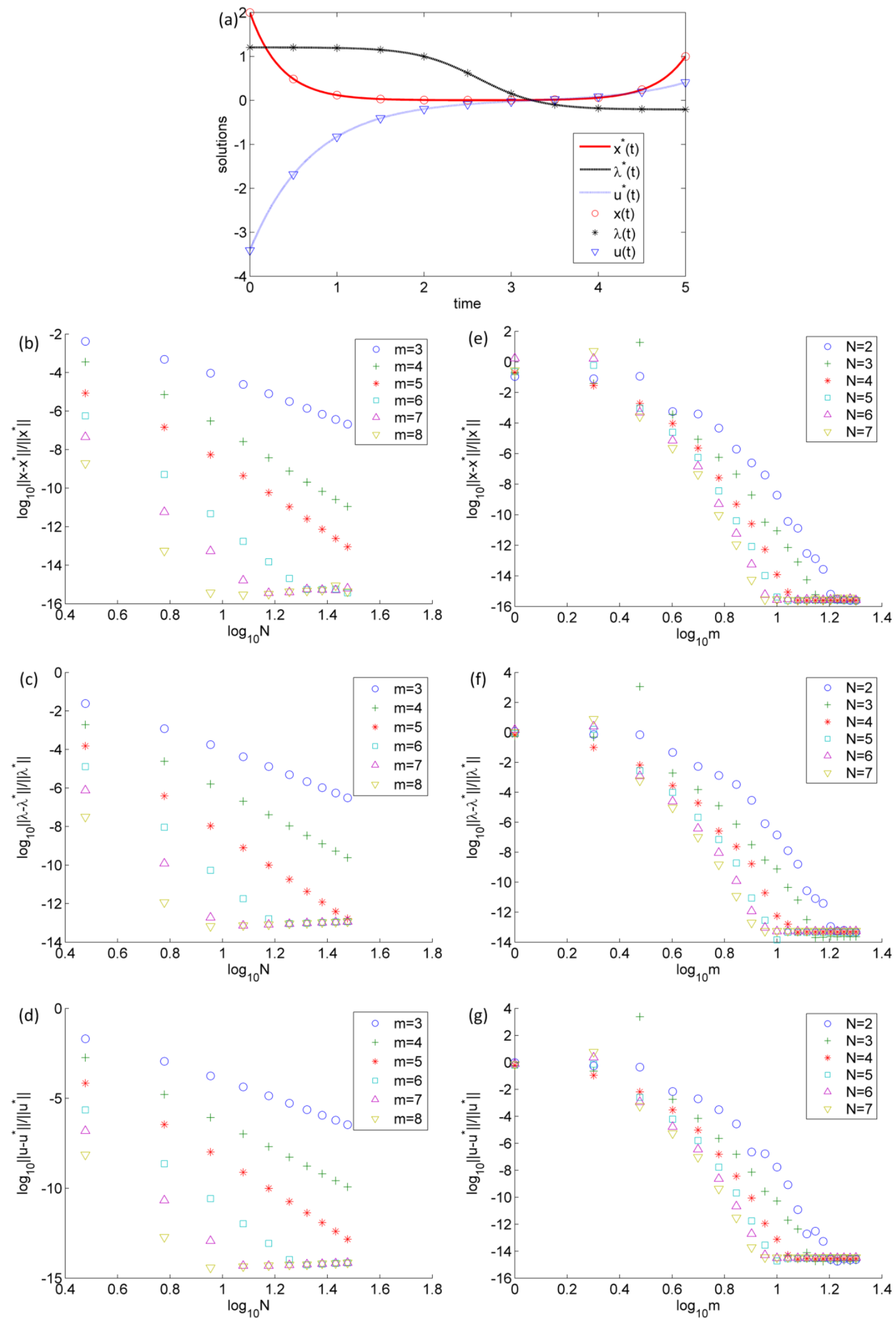
Fig. 2 Numerical results: (a) the optimal solutions with $N=10, m=5$; the relative error with fixed $m$ as $N$ increases: (b) state, (c) costate and (d) control input; the relative error with fixed $N$ as $m$ increases: (e) state (f) costate and (g) control input.

After $N$ and $m$ are chosen to be 10 and 5, respectively, converged solutions are obtained after 11 iterations which take $0.091 \mathrm{~s}$ and the relative error of state equals to $1.90 \mathrm{e}-9$. Numerical solutions along with the analytical solutions are presented in Fig. 2(a). One may find that the obtained solutions have a good agreement with the analytical solutions. Moreover, numerical simulations are carried out for various $N$ with fixed $m$, and various $m$ with fixed $N$. Relative errors of state variables, costate variables and control input which are denoted as $\varepsilon_{x}, \varepsilon_{\lambda}$ and $\varepsilon_{u}$, respectively, are given in Fig. 2(b g). In Fig. 2(b d), $N$ is increased from 3 to 30 with step 3 while $m$ is set to be from 3 to 8 . In Fig. 2(e $\sim$ g), $m$ varies from 1 to 20 with step 1 while $N$ is set to be from 2 to 7 . From Fig. 2(b g), one may find that the relative error decreases quickly as $N$ or $m$ increases. The proposed method shows linear convergence rate when $N$ increases while $m$ is fixed, as can be seen in Fig. 2(b-d). On the other hand, exponent convergence rates are obtained for increasing $m$ with fixed $N$, just the same as direct pseudospetral methods.

As can be seen in Table 1, the performance of the method proposed in this paper outweighs that of the symplectic method proposed in reference [32] in several ways. The rate of convergence of the latter method cannot reach exponent convergence because it is an $h$-mothod. By contrast, the proposed method exhibits exponent convergence and highly accurate numerical solutions can be obtained after few iterations with less time than the symplectic method. In fact, since the proposed method is of sparse and symmetric coefficient matrix and of exponent convergence, the computational costs for the proposed method are less than that of the symplectic method when the same accuracy is required. For even larger $N$ and $m$, the time costs for the proposed method are acceptable because the Jacobian matrix is sparse and symmetric. The advantages of the proposed method are also highlighted when comparing to the direct pseudospectral method. On one hand, the scale of the transformed NLP in direct pseudospectral method increased much quickly than the number of collocation points, which results in high computational costs. On the other hand, optimization solvers for solving the transformed NLP are not required because the optimal control problems have been transformed into a set of nonlinear 
equations. Thus, one may find the proposed method is not only highly efficient but also easily implemented.

Example 2: A Van der Pol problem with free terminal end. The cost functional is given by

$$
J=\int_{0}^{5}\left(x_{1}^{2}+x_{2}^{2}+u^{2}\right) d t
$$

and the dynamic constrains and the initial boundary condition are given by

$$
\left\{\begin{array}{cc}
\dot{x}_{1}=x_{2} & x_{1}(0)=1.5 \\
\dot{x}_{2}=-x_{1}+\left(1-x_{1}^{2}\right) x_{2}+u & x_{2}(0)=1.5
\end{array}\right.
$$

It is noted that the referenced solution of this problem can be obtained by bvp $4 c$ (where the relative error and absolute error are set to be $1.0 e-10$ and 1.0e-12, respectively) in MATLAB. When setting the number of sub-intervals and the degree of approximation polynomial in each sub-interval to 10 and 5, respectively, the converged solution can be obtained as Fig. 3. One may find that obtained solutions agree with referenced solutions very well.
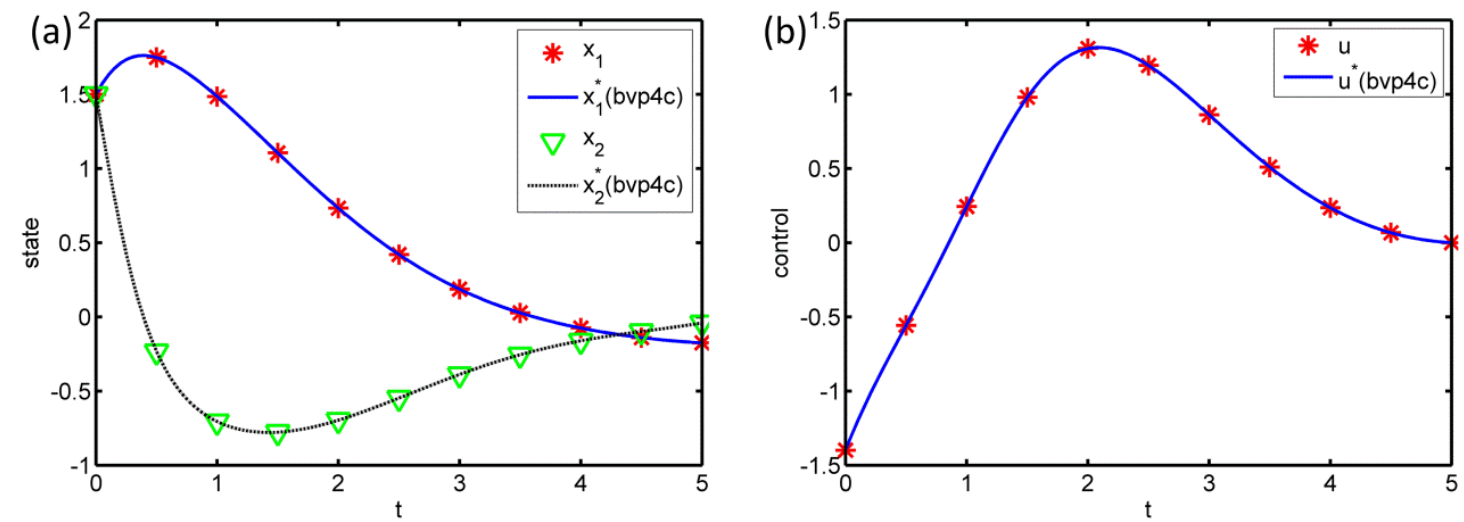

Fig.3 Optimal solutions including (a) state variables and (b) control input

Consider the following three choices of mesh intervals which divide the whole time domain into 10 sub-intervals in dissimilar ways, as shown in Fig. 4. In choice (1),11 interval ends distribute equidistantly in the whole time domain, leading to a regular mesh. In choice (2), there are more sub-intervals near the region $t \in[0.5,2]$, where the trajectory of $x_{2}$ is more complicated, than that far away from this region. In the contrast, sub-intervals distributed near the region $t \in[0.5,2]$ are of small amount in choice (3). 


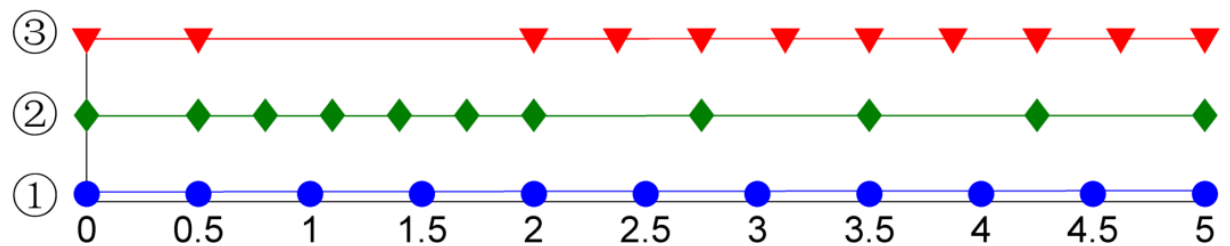

Fig. 4 Three cases of mesh intervals

The given example is solved using each mesh interval given in Fig.4 with the degree of approximation polynomial in each sub-interval fixed to be 3. Comparison of three choices is provided in Table 2 for various parameters. It suggests that adding more sub-intervals near where the trajectory is complicated may lead to high precision and low time cost. As discussed in Section 4, the proposed adaptive procedure may generate more sub-intervals near where the trajectory is complicated. In the following two examples, the adaptive procedure is utilized.

Table 2 Comparison between three choices of mesh interval

\begin{tabular}{cccc}
\hline \hline Mesh interval number & Iterations & $\left\|x_{2}-x_{2}^{*}\right\| /\left\|x_{2}^{*}\right\|$ & CPU times/s \\
\hline (1) Case & 16 & $4.76 e-4$ & 0.1608 \\
(2) Case & 13 & $9.74 e-5$ & 0.1229 \\
(3) Case & 20 & $1.41 e-2$ & 0.1927 \\
\hline \hline
\end{tabular}

Example 3: Consider the following variation of the hyper-sensitive optimal control problem. Minimize the cost functional

$$
J=\frac{1}{2} \int_{0}^{t_{f}}\left(x^{2}+u^{2}\right) d t
$$

subject to the dynamic constraint

$$
\dot{x}=-x+u
$$

and the boundary conditions

$$
x(0)=1.5, x\left(t_{f}\right)=1
$$

where $t_{f}$ is fixed. When $t_{f}$ is sufficiently large, the solution to the hyper-sensitive problems mainly comes down to three stages, which may exhibit a so called "take-off"-"cruise"-"landing" structure. And all of the interesting behaviors take places near the "take-off" and the "landing" region while the solution in the "cruise" region almost remains stationary. Moreover, as the terminal time increases, the "cruise" region takes a great percentage of the whole time domain while the "take-off" region and the 
"landing" region decays or grows exponentially. It is noted that the analytical solution of this problem is available in [27].

In this problem one sets $\mu=1.0 e-6, R=1.2, A=1, B=1.2$, iterMax $=20$. If $t_{f}$ is set to be 1000, and the initial mesh is given as 10 equidistant sub-intervals with the degree of interpolation function to 2 for every sub-interval, then numerical solution for state variables, costate variables and control inputs along with exact solutions are shown in Fig. 5(a) (c), respectively. Iteration histories including the number of sub-intervals $N$, the highest degree of interpolation function of all sub-intervals $m \_$max, number of known variables $v$, the max residual error $e$ as defined in Eq. (77) of all sub-intervals, the relative error of state variables $r$ and computational time cost $t$ in each iteration are given in Table 3. One may find that the numerical results obtained coincide with the analytical solution very well. Moreover, the ends of sub-intervals and the collocation points in each iteration step is given in Fig. 5(d e), respectively. As can be seen in Fig. 5(d e) and Table 3, the solution achieves the demand of accuracy after four iterations with CPU time 1.416s. In the iteration process, the mesh is divided into smaller sub-intervals in the first three iterations, while the degree of interpolation function increases in sub-intervals near two ends in the fourth iteration. In addition, one may find two features form the two figures. First, mesh intervals are only added in the regions near $t=0$ and $t=t_{f}$, while not added in the region $t \in[100,900]$ which is far away from the start and the terminal of the whole time domain. Second, no more mesh intervals are added after the third iteration, collocation points are also added in the regions near $t=0$ and $t=t_{f}$ but are not added in the interior region $t \in[100,900]$. It suggests that the error reduction is achieved where points are needed to capture changes in the trajectory of state variables. As stated formerly, interesting behaviors happen near the "take-off" and the "landing" region, and then numerical solutions of state variable in $[0,10]$ and $[990,1000]$ in each iteration along with the analytical solution of state variable are shown in Fig. 5(f $\sim \mathrm{g})$.

Table 3 Parameters in the iteration history

\begin{tabular}{cccccccccc}
\hline \hline \multirow{2}{*}{ Iterations } & $N$ & m_max & $v$ & $e$ & $R$ & \multicolumn{2}{c}{$t / \mathrm{s}$} \\
& & & & & & & & & \\
Remeshing & Solving & Total \\
\hline initial mesh & 10 & 2 & 51 & $2.499 \mathrm{e} 3$ & $4.989 \mathrm{e}-1$ & - & 0.011 & 0.011 \\
1 & 32 & 9 & 195 & $1.689 \mathrm{e} 2$ & $5.100 \mathrm{e}-3$ & 0.003 & 0.033 & 0.036 \\
2 & 48 & 9 & 323 & $1.023 \mathrm{e}-1$ & $3.868 \mathrm{e}-4$ & 0.004 & 0.056 & 0.061 \\
3 & 54 & 9 & 489 & $3.905 \mathrm{e}-4$ & $8.244 \mathrm{e}-8$ & 0.010 & 0.156 & 0.166 \\
4 & 54 & 9 & 545 & $6.200 \mathrm{e}-7 \quad 1.017 \mathrm{e}-10$ & 0.009 & 1.041 & 1.050
\end{tabular}


(a)

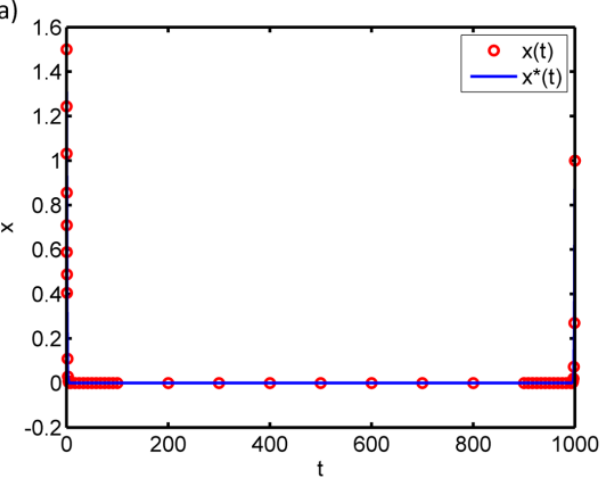

(b)
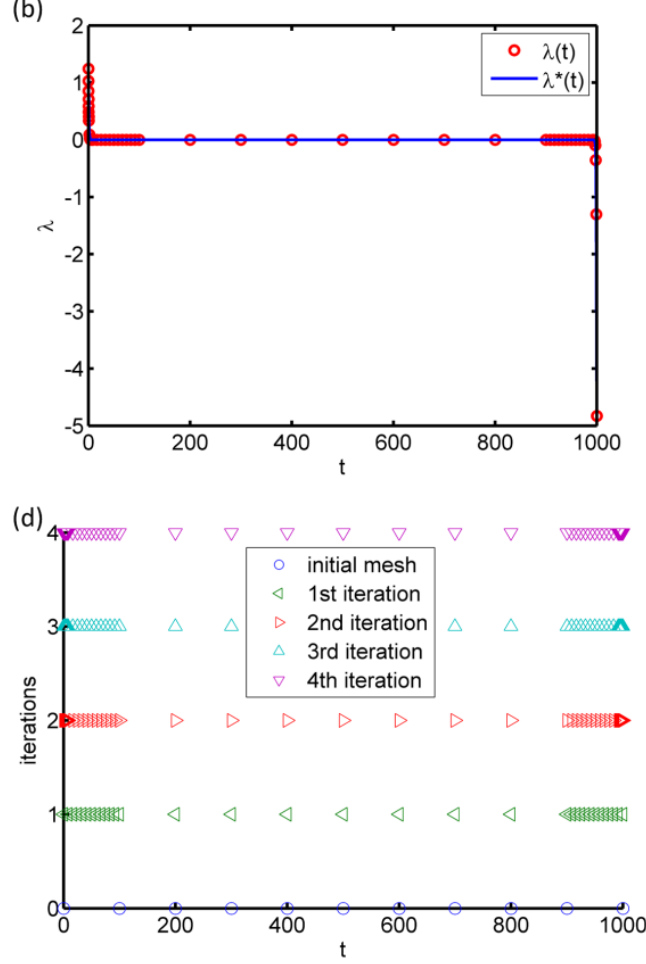

(f)

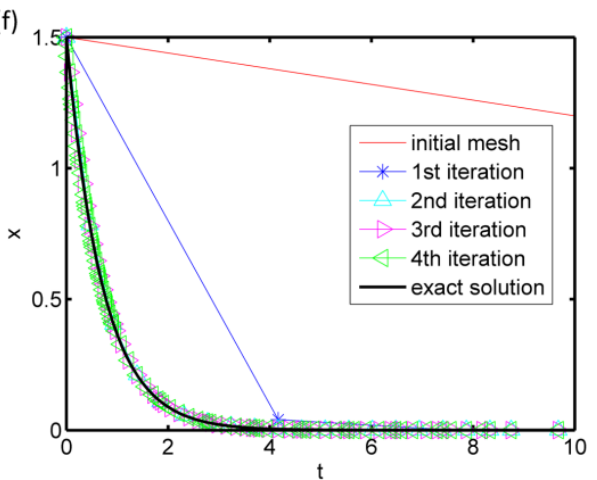

(c)
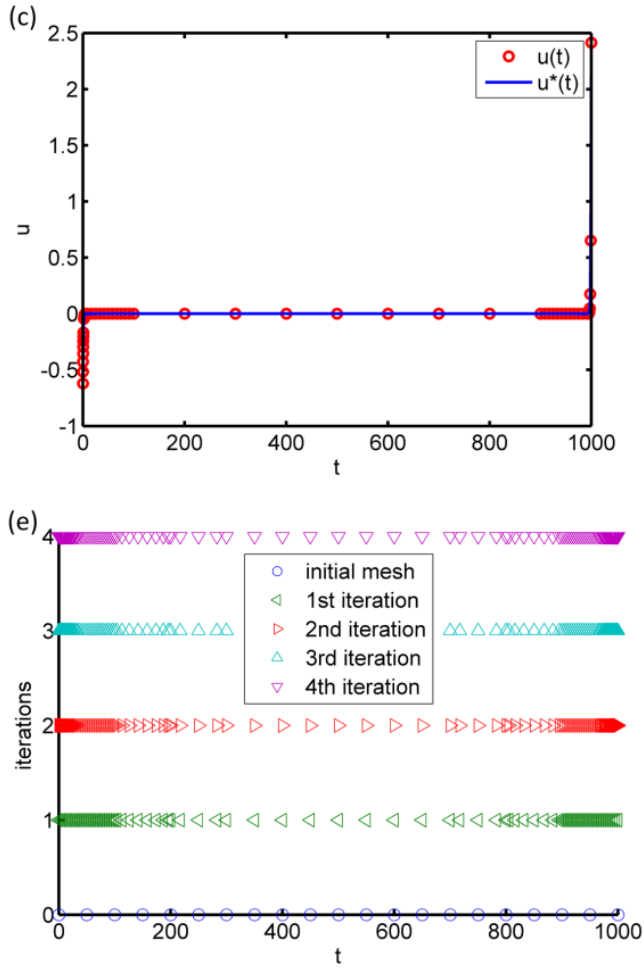

(g)

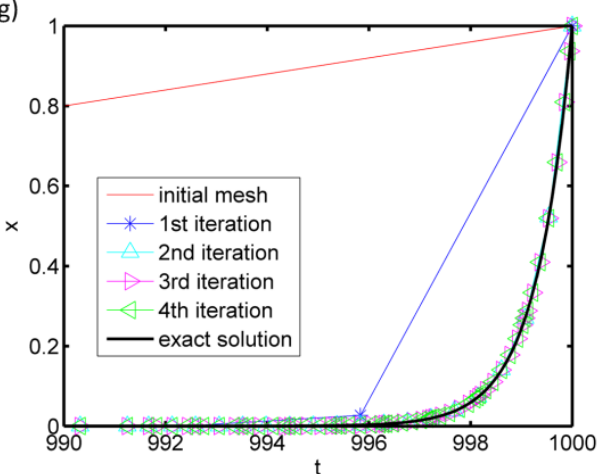


Fig. 5 Optimal solutions of (a) state variable, (b) costate variable and (c) control input along with exact solutions. Time history: (d) mesh intervals and (e) collocation points. Time history of optimal solution of state variable in region (f) $[0,10]$ and (g) $[990,1000]$.

Example 4: Consider a spacecraft or satellite subject to a central gravity field of earth. In a coordinate system which is rotating along a circular orbit around earth at a constant angular velocity, the non-dimensional motion equations of this spacecraft can be written as

$$
\begin{gathered}
\ddot{x}-2 \dot{y}+(1+x)\left(\frac{1}{r^{3}}-1\right)=u_{x} \\
\ddot{y}+2 \dot{x}+y\left(\frac{1}{r^{3}}-1\right)=u_{y} \\
\ddot{z}+\frac{1}{r^{3}} z=u_{z}
\end{gathered}
$$

where

$$
r^{2}=(1+x)^{2}+y^{2}+z^{2}
$$

The symbols $x, y$ and $z$ are the positions of the spacecraft which represent radial, tangential and normal displacements from the origin of the rotating frame, while the dot represent derivation with respect to time. And $u_{x}, u_{y}, u_{z}$ denote the control forces in the $x, y$ and $z$ directions, respectively.

In the rendezvous process, the spacecraft is transferred from the initial state to the goal state at the terminal time $t_{f}=1$. In this example, the boundary conditions are given as

$$
\begin{gathered}
x(0)=y(0)=y(0)=0.3 ; \dot{x}(0)=\dot{y}(0)=\dot{z}(0)=0.2 \\
x(1)=y(1)=y(1)=0 ; \dot{x}(1)=\dot{y}(1)=\dot{z}(1)=0
\end{gathered}
$$

The objective of this problem is minimize the fuel cost and then the performance index can be defined as follows

$$
J=\frac{1}{2} \int_{0}^{1}\left(u_{x}^{2}+u_{y}^{2}+u_{z}^{2}\right) d t
$$

In this problem one sets $\mu=1.0 e-8, R=1.1, A=1, B=1.2$, iterMax $=20$. The initial mesh is given as four equidistant sub-intervals with a 4-order interpolation function applied to each sub-interval. The solutions are eventually obtained after two refinement iterations with CPU time to be 
1.402s. The solutions of the position and velocity of the spacecraft are present in Fig. 6(a b), respectively. Moreover, the ends of sub-intervals and the collocation points in each iteration are shown in Fig. 6(c d). Values such as the cost functional $J$, the number of sub-intervals $N$, and the highest degree of interpolation functions all of sub-intervals $m \_$max, the number of unknown variables $v$ and computational time cost $t$ in each iteration are given in Table 4. As can be seen in Table 4, the precision of the solution is improved quickly as the iteration goes on. One may find that in region $[0.75,1]$ mesh intervals are not added but the number of collocation points is increased, which implies the relative curvature of trajectory of the each state variable is small in this region.

Table 4 Parameters in the iteration history

\begin{tabular}{cccccccc}
\hline \hline \multirow{2}{*}{ Iterations } & \multirow{2}{*}{$J$} & $N$ & m_max & $v$ & \multicolumn{3}{c}{$t / \mathrm{s}$} \\
\cline { 7 - 9 } & & & & & Remeshing & Sloving & Total \\
initial mesh & $\mathbf{3 . 3 8 6 0 6 5 7 9 0 0 1 4 5 6 5}$ & 4 & 4 & 222 & - & 0.022 & 0.022 \\
1 & $\mathbf{3 . 3 8 6 8 2 0 0 1 3 7 2 4 0 9 4}$ & 20 & 10 & 1158 & 0.003 & 0.303 & 0.306 \\
2 & $\mathbf{3 . 3 8 6 8 2 0 4 0 1 7 5 2 6 9 9}$ & 20 & 10 & 1842 & 0.006 & 1.051 & 1.058 \\
3(final check) & - & 20 & 10 & 1842 & 0.006 & - & 0.006 \\
\hline \hline
\end{tabular}

(a)

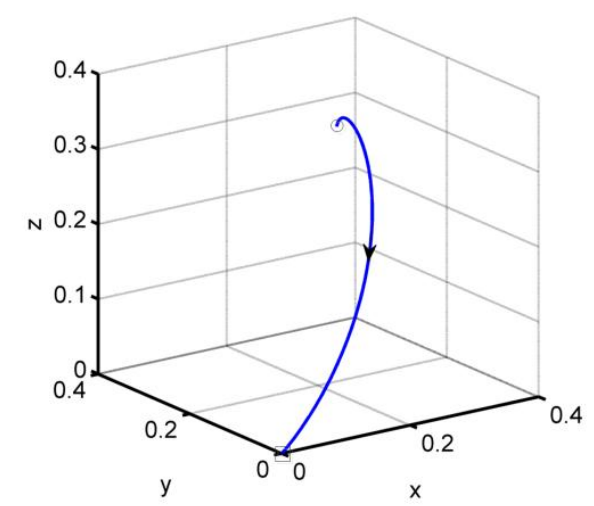

(c)

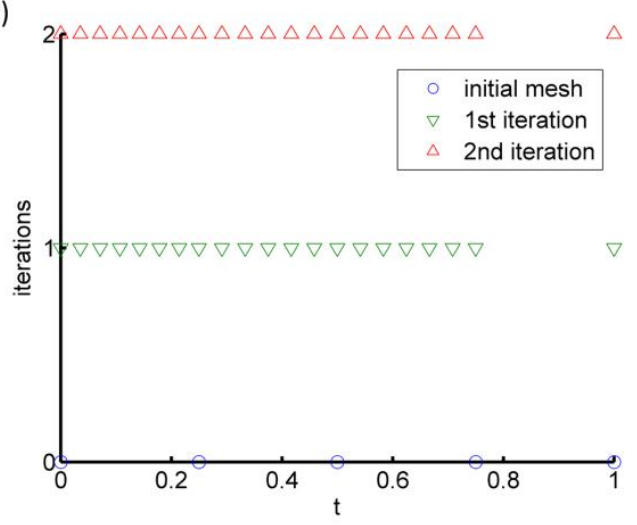

(b)

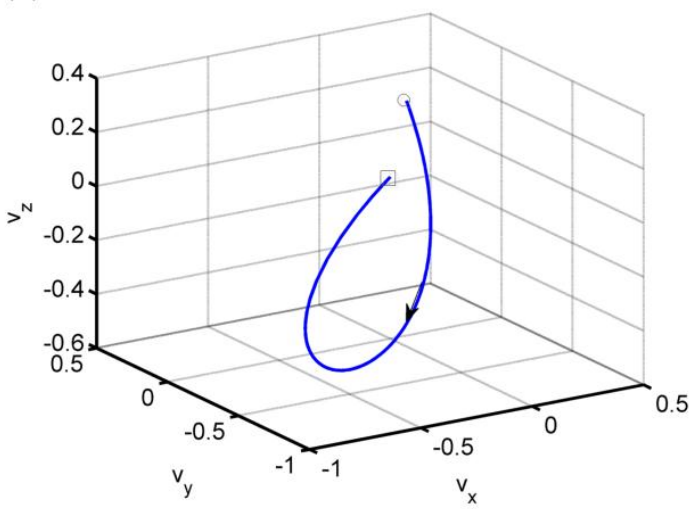

(d)

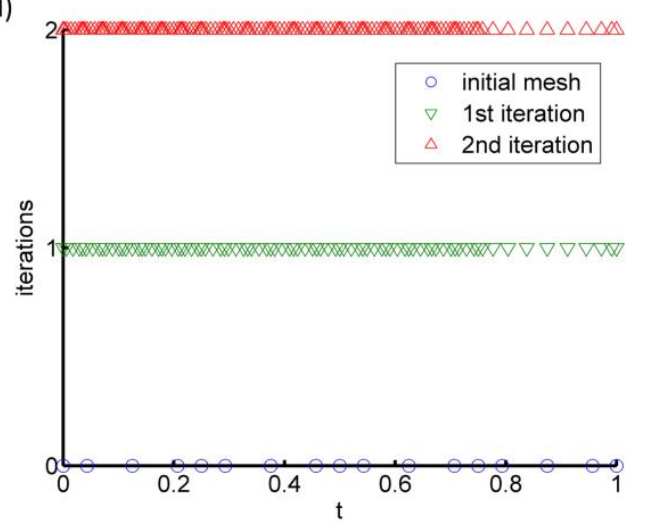

Fig. 6 Optimal trajectory of (a) position and (b) velocity in three-dimension. Time history of (c) mesh intervals and (d) collocation points. 
Example 5: Consider a two-body tethered satellite system shown in Fig. 7, where the mother satellite ( $\left.S_{1}\right)$ mass is far bigger than the subsatellite $\left(S_{2}\right)$ mass. The dynamic equations of the system can be expressed as

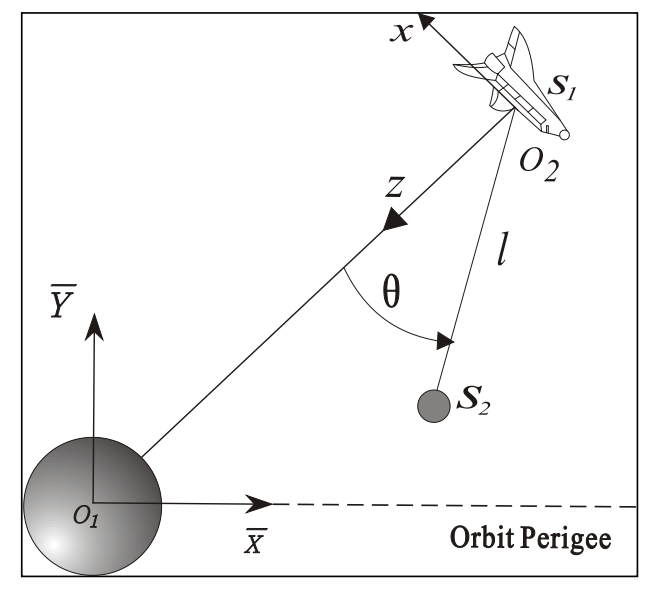

Fig. 7 Motion of a two-body tethered satellite system project to the plane of system orbit.

$$
\left\{\begin{array}{c}
\ddot{\theta}+2(\dot{\theta}+1)(\dot{\Lambda} / \Lambda)+3 \sin \theta \cos \theta=0 \\
\ddot{\Lambda}-\Lambda\left[(\dot{\theta}+1)^{2}+3 \cos ^{2} \theta-1\right]=u
\end{array}\right.
$$

where $\theta$ describes the pitching movement of the tether, $\Lambda$ is the ratio of the current length of the tether $L$ to a referenced length $L_{r e f}$ and the dot represents the derivative with respect to the non-dimensional time variable $v$. By replacing $\theta$ and $\Lambda$ by $x_{1}$ and $x_{3}$, respectively, Eq.(101) can be transformed into a standard form as

$$
\left\{\begin{array}{c}
\dot{x}_{1}=x_{2} \\
\dot{x}_{2}=-2\left(x_{2}+1\right)\left(x_{4} / x_{3}\right)-3 \sin x_{1} \cos x_{1} \\
\dot{x}_{3}=x_{4} \\
\dot{x}_{4}=x_{3}\left[\left(x_{2}+1\right)^{2}+3 \cos x_{1}-1\right]-u
\end{array}\right.
$$

Three cases with different initial states and the same final state are given to simulate the deployment of the tethered satellite system

$$
\begin{gathered}
\left\{\begin{array}{c}
\text { case } 1: x_{1}(0)=0, x_{2}(0)=0, x_{3}(0)=0.1, x_{4}(0)=0.2 \\
\text { case 2: } x_{1}(0)=-0.872, x_{2}(0)=0, x_{3}(0)=0.3, x_{4}(0)=0 \\
\text { case 3: } x_{1}(0)=1, x_{2}(0)=-0.53, x_{3}(0)=0.1, x_{4}(0)=0.3
\end{array}\right. \\
\qquad x_{1}\left(t_{f}\right)=0, x_{2}\left(t_{f}\right)=0, x_{3}\left(t_{f}\right)=1, x_{4}\left(t_{f}\right)=0
\end{gathered}
$$

where $t_{f}$ is set to be 6 and the objective is to minimize the cost functional which is expressed as 


$$
J=\frac{1}{2} \int_{0}^{t_{f}} 10 x_{1}^{2}+5 x_{2}^{2}+10\left(x_{3}^{2}-1\right)^{2}+x_{4}^{2}+(u-3)^{2} d v
$$

When the number of sub-intervals and the degree of interpolation functions applied in each sub-interval are set to be 10 and 10, respectively, converged solutions of each case can be obtained as Fig. 8(a e) and the cost functionals of three cases are calculated to be $12.61913,10.51679$ and 17.37296, respectively.

(a)

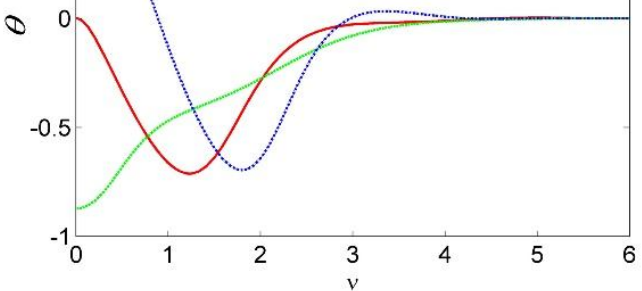

(c)

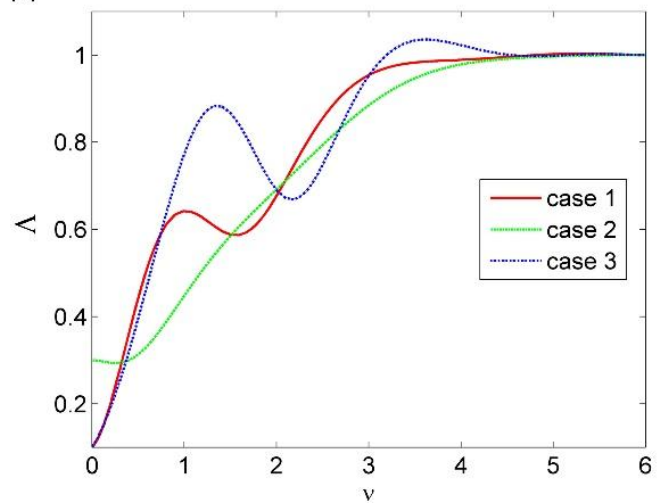

(b)

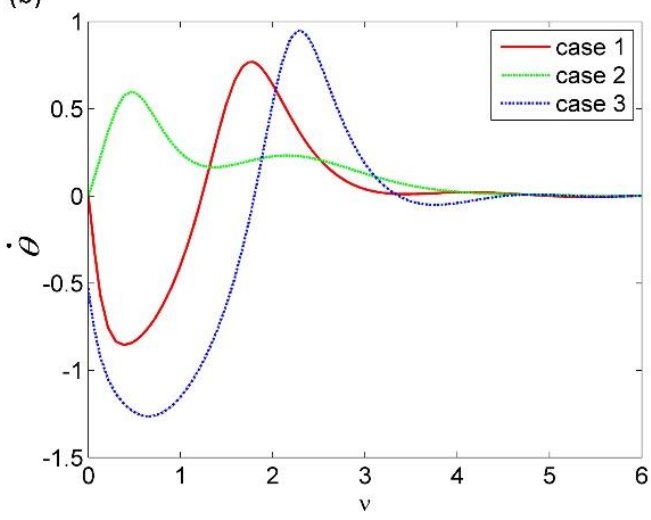

(d)

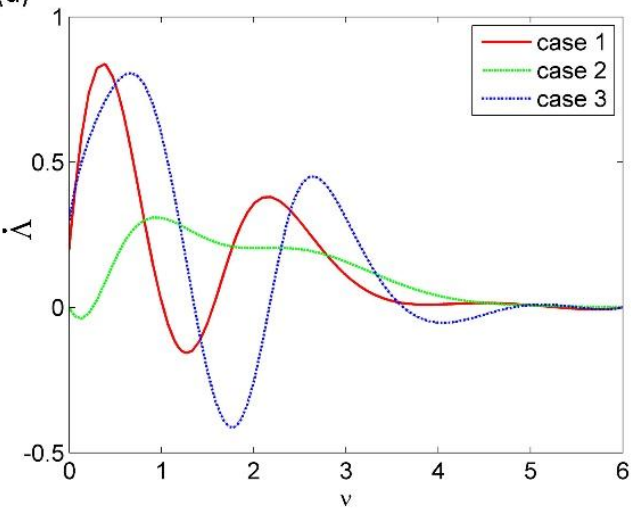

(e)

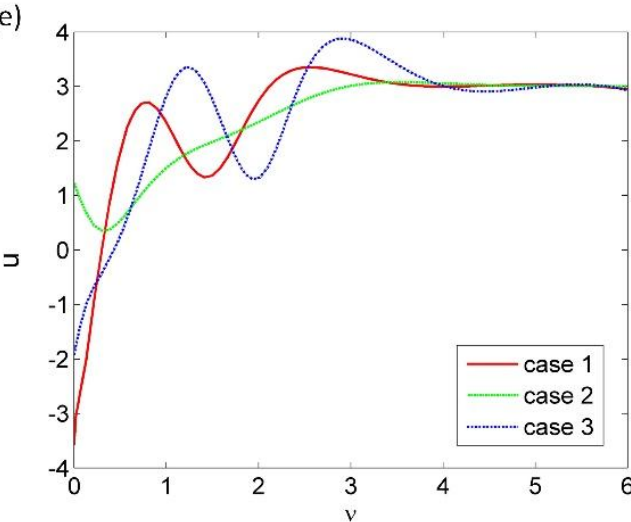

Fig. 8 Optimal solutions of (a) $\theta$, (b) $\dot{\theta}$,(c) $\Lambda$,(d) $\dot{\Lambda}$ and (e) $u$. 
To exhibit the motion of subsatellite projected to the plane of system orbit, we define a nondimensional coordinate system $\left(x_{e}, y_{e}\right)$ as in Eq. (106) and the mother satellite is fixed at the original point. Thus, trajectories of the subsatellite in three cases are plotted in Fig. $9(\mathrm{a} \sim \mathrm{c})$, respectively.

$$
x_{e}=\Lambda \sin \theta, \mathrm{y}_{e}=\Lambda \cos \theta
$$

(a)

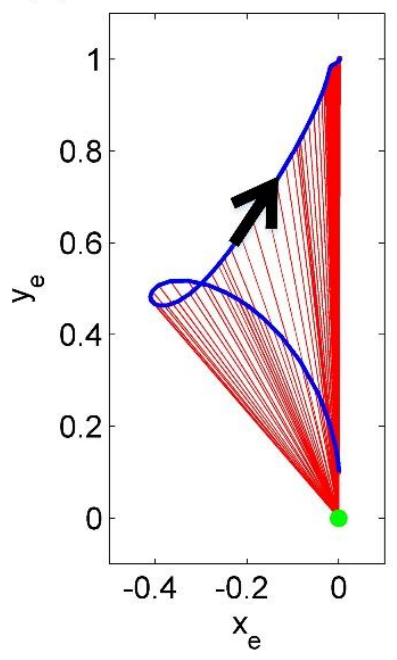

(b)

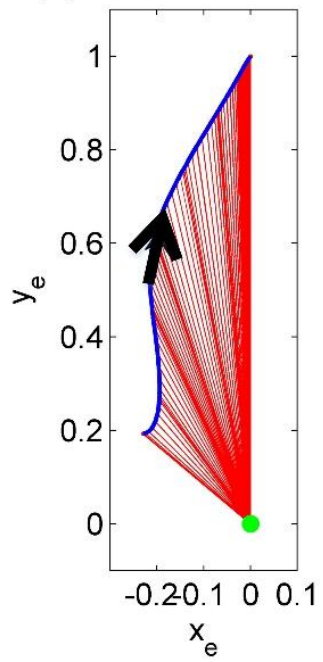

(c)

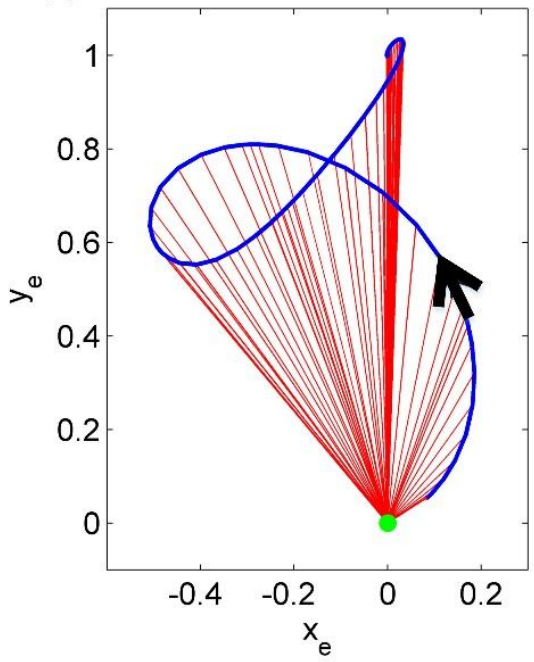

Fig. 9 The trajectory of the subsatellite in (a) case1, (b) case 2 and (c) case 3.

\section{Conclusions}

An $h p$ symplectic pseudospectral method based on the dual variational method for solving optimal control problem is proposed in this paper. The main conclusions can be summarized as:

(1) The proposed symplectic pseudospectral method can achieve arbitrary accuracy by increasing the number (denotes by $N$ ) of sub-interval and/or increasing the number of collocation points (denotes by $m$ ) in each sub-intervals. The proposed method, on one hand, exhibits exponent convergence rate when $m$ is increasing with fixed $N$; on the other hand, exhibits linear convergence rate when $N$ is increasing with fixed $m$.

(2) An $h p$ adaptive method combined with the sympletic pseudospectral method can obtain a fine solution on a reasonable mesh, which avoids using either too many sub-intervals or too high degree of approximation polynomial, leading to a high accuracy and low computational cost.

(3) The adaptive symplectic pseudospectral method is applied to five examples, highlighting the high efficiency and high precision of the proposed method. 


\section{Acknowledgments}

The authors are grateful for the financial support of the National Science Foundation of China (11472069, 11432010); the Project Funded by China Postdoctoral Science Foundation (2014M550155, 2015T80245); the Dalian Science and Technology Project (2015A11GX037); the Fundamental Research Funds for the Central Universities (DUT16LK21).

\section{Appendix A}

Detailed expression of items in Eqs. (42) (43) are

$$
\begin{aligned}
& \mathbf{K}_{11}^{(j)}=\left(\mathbf{K}_{00}^{x x}\right)^{(j)} \\
& \left.\mathbf{K}_{12}^{(j)}=\left[\begin{array}{llll}
\left(\mathbf{K}_{01}^{x x}\right)^{(j)} & \left(\mathbf{K}_{02}^{x x}\right)^{(j)} & \cdots & \left(\mathbf{K}_{0(N-1)}^{x x}\right.
\end{array}\right)^{(j)}\right] \\
& \mathbf{K}_{13}^{(j)}=\left(\mathbf{K}_{0 N}^{x x}\right)^{(j)} \\
& \mathbf{K}_{14}^{(j)}=\left[\begin{array}{llll}
\left(\mathbf{K}_{00}^{x \lambda}\right)^{(j)} & \left(\mathbf{K}_{01}^{x \lambda}\right)^{(j)} & \cdots & \left(\mathbf{K}_{0 N}^{x \lambda}\right)^{(j)}
\end{array}\right] \\
& \mathbf{K}_{21}^{(j)}=\left(\mathbf{K}_{12}^{(j)}\right)^{\mathrm{T}} \\
& \mathbf{K}_{22}^{(j)}=\left[\begin{array}{cccc}
\left(\mathbf{K}_{11}^{x x}\right)^{(j)} & \left(\mathbf{K}_{12}^{x x}\right)^{(j)} & \cdots & \left(\mathbf{K}_{(N-1)(N-1)}^{x x}\right)^{(j)} \\
\left(\mathbf{K}_{21}^{x x}\right)^{(j)} & \left(\mathbf{K}_{22}^{x x}\right)^{(j)} & \cdots & \left(\mathbf{K}_{(N-1)(N-1)}^{x x}\right)^{(j)} \\
\vdots & \vdots & \ddots & \vdots \\
\left(\mathbf{K}_{(N-1) 1}^{x x}\right)^{(j)} & \left(\mathbf{K}_{(N-1) 2}^{x x}\right)^{(j)} & \cdots & \left(\mathbf{K}_{(N-1)(N-1)}^{x x}\right)^{(j)}
\end{array}\right] \\
& \mathbf{K}_{23}^{(j)}=\left[\left(\left(\mathbf{K}_{1(N-1)}^{x x}\right)^{(j)}\right)^{\mathrm{T}} \quad\left(\left(\mathbf{K}_{2(N-1)}^{x x}\right)^{(j)}\right)^{\mathrm{T}} \cdots\left(\left(\mathbf{K}_{(N-1)(N-1)}^{x x}\right)^{(j)}\right)^{\mathrm{T}}\right]^{\mathrm{T}} \\
& \mathbf{K}_{24}^{(j)}=\left[\begin{array}{cccc}
\left(\mathbf{K}_{10}^{x \lambda}\right)^{(j)} & \left(\mathbf{K}_{11}^{x \lambda}\right)^{(j)} & \cdots & \left(\mathbf{K}_{1 N}^{x \lambda}\right)^{(j)} \\
\left(\mathbf{K}_{20}^{x \lambda}\right)^{(j)} & \left(\mathbf{K}_{21}^{x \lambda}\right)^{(j)} & \cdots & \left(\mathbf{K}_{2 N}^{x \lambda}\right)^{(j)} \\
\vdots & \vdots & \ddots & \vdots \\
\left(\mathbf{K}_{(N-1) 0}^{x \lambda}\right)^{(j)} & \left(\mathbf{K}_{(N-1) 1}^{x \lambda}\right)^{(j)} & \cdots & \left(\mathbf{K}_{(N-1) N}^{x \lambda}\right)^{(j)}
\end{array}\right] \\
& \mathbf{K}_{31}^{(j)}=\left(\mathbf{K}_{13}^{(j)}\right)^{\mathrm{T}}
\end{aligned}
$$




$$
\begin{aligned}
& \mathbf{K}_{32}^{(j)}=\left(\mathbf{K}_{23}^{(j)}\right)^{\mathrm{T}} \\
& \mathbf{K}_{33}^{(j)}=\left(\mathbf{K}_{N N}^{x x}\right)^{(j)} \\
& \mathbf{K}_{34}^{(j)}=\left[\begin{array}{llll}
\left(\mathbf{K}_{N 0}^{x \lambda}\right)^{(j)} & \left(\mathbf{K}_{N 1}^{x \lambda}\right)^{(j)} & \cdots & \left(\mathbf{K}_{N N}^{x \lambda}\right)^{(j)}
\end{array}\right] \\
& \mathbf{K}_{41}^{(j)}=\left(\mathbf{K}_{14}^{(j)}\right)^{\mathrm{T}} \\
& \mathbf{K}_{42}^{(j)}=\left(\mathbf{K}_{24}^{(j)}\right)^{\mathrm{T}} \\
& \mathbf{K}_{43}^{(j)}=\left(\mathbf{K}_{34}^{(j)}\right)^{\mathrm{T}} \\
& \mathbf{K}_{44}^{(j)}=\left[\begin{array}{cccc}
\left(\mathbf{K}_{00}^{\lambda \lambda}\right)^{(j)} & \left(\mathbf{K}_{01}^{\lambda \lambda}\right)^{(j)} & \cdots & \left(\mathbf{K}_{0 N}^{\lambda \lambda}\right)^{(j)} \\
\left(\mathbf{K}_{10}^{\lambda \lambda}\right)^{(j)} & \left(\mathbf{K}_{11}^{\lambda \lambda}\right)^{(j)} & \cdots & \left(\mathbf{K}_{1 N}^{\lambda \lambda}\right)^{(j)} \\
\vdots & \vdots & \ddots & \vdots \\
\left(\mathbf{K}_{N 0}^{\lambda \lambda}\right)^{(j)} & \left(\mathbf{K}_{N 1}^{\lambda \lambda}\right)^{(j)} & \cdots & \left(\mathbf{K}_{N N}^{\lambda \lambda}\right)^{(j)}
\end{array}\right] \\
& \mathbf{F}_{1}^{(j)}=\left(\mathbf{f}_{0}^{x}\right)^{(j)} \\
& \mathbf{F}_{2}^{(j)}=\left[\left(\left(\mathbf{f}_{1}^{x}\right)^{(j)}\right)^{\mathrm{T}},\left(\left(\mathbf{f}_{2}^{x}\right)^{(j)}\right)^{\mathrm{T}}, \ldots,\left(\left(\mathbf{f}_{N-1}^{x}\right)^{(j)}\right)^{\mathrm{T}}\right]^{\mathrm{T}} \\
& \mathbf{F}_{3}^{(j)}=\left(\mathbf{f}_{N}^{x}\right)^{(j)} \\
& \mathbf{F}_{4}^{(j)}=\left[\left(\left(\mathbf{f}_{0}^{\lambda}\right)^{(j)}\right)^{\mathrm{T}},\left(\left(\mathbf{f}_{1}^{\lambda}\right)^{(j)}\right)^{\mathrm{T}}, \ldots,\left(\left(\mathbf{f}_{N}^{\lambda}\right)^{(j)}\right)^{\mathrm{T}}\right]^{\mathrm{T}}
\end{aligned}
$$

\section{References}

[1]. Upreti SR. A new robust technique for optimal control of chemical engineering processes. Comput Chem Eng 2004;28(8):1325-1336.

[2]. Logist F, Houska B, Diehl M. Robust multi-objective optimal control of uncertain (bio)chemical processes. Chem Eng Sci 2011;66(20):4670-4682.

[3]. Costanza V, Rivadeneira PS. Hamiltonian two-degrees-of-freedom control of chemical reactors. Optim Contr Appl Met 2011;32(3):350-368. 
[4]. Karimi HR, Lohmann B. Haar Wavelet-based robust optimal control for vibration reduction of vehicle engine-body system. Electr Eng 2007;89(6):469-478.

[5]. Hanagud S, Obal MW, Calise AJ. Optimal vibration control by use of piezoceramic sensors and actuators. J Guid Control Dynam 1992;15(5):1199-1206.

[6]. Uno Y, Kawato M, Suzuki R. Formation and control of optimal trajectory in human multijoint arm movement. Biol Cybern 1989;61(2):89-101.

[7]. Schaal S, Atkeson CG. Learning Control in Robotics. IEEE Robot Autom Mag 2010; 17(2):20-29.

[8]. Bergerman M, Xu Y. Optimal control sequence for underactuated manipulators. Proceedings of IEEE International Conference on Robotics and Automation; 1996 Apr 22-28; Minneapolis, USA. 3714-3719.

[9]. Hermant A. Optimal control of the atmospheric reentry of a space shuttle by an homotopy method. Optim Contr Appl Met 2011;32(6):627-646.

[10].Lee T, Mcclamroch NH, Leok M. A combinatorial optimal control problem for spacecraft formation reconfiguration; Proceedings of the IEEE Conference on Decision \& Control. 2007:5370-5375.

[11].Han D, Balakrishnan SN. State-constrained agile missile control with adaptive-critic-based neural networks. Ieee T Contr Syst T 2002;10(4):481-489.

[12].Fumenti F, Circi C, Romagnoli D. Collocation points distributions for optimal spacecraft trajectories. Commun Nonlinear Sci 2013;18(3):710-727.

[13]. Stryk OV, Bulirsch R. Direct and indirect methods for trajectory optimization. Ann Oper Res 1992;37(1):357-373.

[14]. Bryson AE, Ho YC. Applied optimal control. Hemisphere/Wiley; 1975.

[15].Holsapple R, Venkataraman R, Doman D. A modified simple shooting method for solving two-point boundary-value problems. Nasa Sti/recon Technical Report N 2002;3(6):2783-2790.

[16].Lastman GJ. A shooting method for solving two-point boundary-value problems arising from non-singular bang-bang optimal control problems. Int J Control 1978;27(4):513-524.

[17]. Fotouhi CR, Szyszkowski W. A numerical approach for time-optimal control of double arms robot. Proceedings of the 4th IEEE Conference on Control Applications. 1995:1128-1133. 
[18].Fabien BC. Numerical solution of constrained optimal control problems with parameters. Appl Math Comput 1996;80(1):43-62.

[19].Park C, Scheeres DJ. Determination of optimal feedback terminal controllers for general boundary conditions using generating functions. Automatica 2006;42(5):869-875.

[20]. Marzban HR, Hoseini SM. A composite Chebyshev finite difference method for nonlinear optimal control problems. Commun Nonlinear Sci 2013;18(6):1347-1361.

[21].Garg D, Patterson MA, Hager WW. A unified framework for the numerical solution of optimal control problems using pseudospectral methods. Automatica 2010;46(11):1843-1851.

[22].Fahroo F, Ross IM. Direct trajectory optimization by a chebyshev pseudospectral method. J Guid Control Dynam 2002;25(1):160-166.

[23]. Benson DA, Huntington GT, Thorvaldsen TP, Rao AV. Direct trajectory optimization and costate estimation via an orthogonal collocation method. J Guid Control Dynam 2006;29(6):1435-1439.

[24].Darby CL, Hager WW, Rao AV. An hp - adaptive pseudospectral method for solving optimal control problems. Optim Contr Appl Met 2011;32(4):476-502.

[25].Garg D, Patterson MA, Francolin C, Darby CL, Huntington GT, Hager WW, et al. Direct trajectory optimization and costate estimation of finite-horizon and infinite-horizon optimal control problems using a radau pseudospectral method. Comput Optim Appl 2011;49(2):335-358.

[26].Rao AV, Benson DA, Darby C, Patterson MA, Francolin C, Sanders I, et al. Algorithm 902: GPOPS, a matlab software for solving multiple-phase optimal control problems using the gauss pseudospectral method. Acm T Math Software 2010;37(2):163-172.

[27].Darby CL. hp-Pseudospectral method for solving continuous-time nonlinear optimal control problems. PhD thesis, Department of Mechanical and Aerospace Engineering, University of Florida, 2011.

[28].Patterson MA, Hager WW, Rao AV. A ph mesh refinement method for optimal control. Optim Contr Appl Met 2014;36:398-421.

[29].Garg D, Hager WW, Rao AV. Pseudospectral methods for solving infinite-horizon optimal control problems. Automatica 2011;47(47):829-837.

[30].Leyendecker S, Ober-Blöbaum S, Marsden JE, Ortiz M. Discrete mechanics and optimal control for constrained systems. Optim Contr Appl Met 2010;31(6):505-528. 
[31]. Arnold VI. Mathematical Methods of Classical Mechanics. Springer New York, 1978.

[32].Peng H, Gao Q, Wu Z, Zhong W. Symplectic approaches for solving two-point boundary-value problems. J Guid Control Dynam 2012;35(2):653-658.

[33].Peng H, Gao Q, Wu Z, Zhong W. Symplectic adaptive algorithm for solving nonlinear two-point boundary value problems in Astrodynamics. Celest Mech Dyn Astr 2011;110(4):319-342.

[34].Peng H, Gao Q, Wu Z, Zhong W. Symplectic algorithms with mesh refinement for a hypersensitive optimal control problem. Int J Comput Math 2014;92(11):1-17.

[35]. Li M, Peng H, Wu Z. Symplectic irregular interpolation algorithms for optimal control problems. Int J Comput Methods 2015;12(6):1-26.

[36].Li M, Peng H, Zhong W. A symplectic sequence iteration approach for nonlinear optimal control problems with state-control constraints. J Frankl Inst 2015;352(6):2381-2406.

[37].Hager WW, Rao AV, Tsiotras P, Darby CL, Betts JT, Becerra VM, et al. Direct trajectory optimization using a variable low-order adaptive pseudospectral method. J Spacecraft Rockets $2011 ; 48(3): 433-445$.

[38]. Gui W, BabuKa I. The h, p and h-p versions of the finite element method in 1 dimension. part I. the error analysis of the p-version. Numer Math 1985;49(6):577-612.

[39]. Gui W, BabuKa I. The h, p and h-p versions of the finite element method in 1 dimension. part II. the error analysis of the $h$ and h-p versions. Numer Math 1986;49(6):613-657.

[40]. Gui W, BabuKa I. The h, p and h-p versions of the finite element method in 1 dimension - part III. the adaptive h-p version. Numer Math 1986;49(6):659-683.

[41].Zienkiewicz OC, Taylor RL, Zhu JZ. The Finite Element Method: Its Basis and Fundamentals. Butterworth-Heinemann, 2005.

[42]. Shen J, Tang T, Wang L. Spectral methods: algorithms, analysis and applications. Springer; 2011.

[43]. Trefethen LN. Spectral methods in MATLAB. Philadelphia: SIAM; 2000

[44]. Steindl A, Troger, H. Optimal control of deployment of a tethered subsatellite. Nonlinear Dynam 2003;31(3): 257-274. 